\title{
Form factors, thermal states and modular structures
}

\author{
Max R. Niedermaier \\ Max-Planck-Institut fïr Gravitationsphysik (Albert-Einstein-Institut), Schlaatzweg I, \\ D-14473 Potsdam, Germany
}

Received 27 November 1997: accepted 4 February 1998

\begin{abstract}
Form factor sequences of an integrable QFT can be defined axiomatically as solutions of a system of recursive functional equations, known as "form factor equations". We show that their solution can be replaced with the study of the representation theory of a novel algebra $\mathcal{F}(S)$. It is associated with a given two-particle $S$-matrix and has the following features: (i) It contains a double TTS algebra as a subalgebra. (ii) Form factors arise as thermal vector states over $\mathcal{F}(S)$ of temperature $1 / 2 \pi$. The thermal ground states are in correspondence to the local operators of the QFT. (iii) The underlying 'finite temperature structure' is indirectly related to the "Unruh effect" in Rindler space-time. In $\mathcal{F}(S)$ it is manifest through modular structures $(j, \delta)$ in the sense of algebraic QFT, which can be implemented explicitly in terms of the TTS generators. (C) 1998 Elsevier Science B.V.
\end{abstract}

PACS: 11.10.Kk; 11.15.Tk; 04.70.Dy

Keywords: Integrable quantum field theory; Form factor approach; Unruh effect

\section{Introduction and survey}

The form factor approach to integrable quantum field theories (QFTs) has several remarkable features. First it yields a complete ('non-perturbative') definition of an integrable QFT, independent of any Lagrangian description. Rather, the QFT is described in terms of "form factors", which arise as elements of sequences of tensor valued meromorphic functions, solving a recursive set of functional equations. Second, it provides a powerful non-perturbative solution technique, yielding results difficult or impossible to obtain otherwise. This is especially relevant when the QFT in question has an independent description as, say, the continuum limit of some lattice system. Third, no regularization and renormalization is necessary to obtain genuine QFT quantities. From the viewpoint of Haag's theorem the last property hints at the existence of an underlying 
algebraic structure with a controllable representation theory, in the sense that the representations relevant for the specification of an interacting QFT can be described explicitly (unlike the situation for the canonical commutation relations).

\section{I.I. Form factor sequences in integrable QFTs}

The algebraic analysis of form factors in an integrable QFT is usually done in terms of the Zamolodchikov-Faddeev (ZF) algebra. Originally the ZF algebra was invented to give a concise description of all the $S$-matrix elements of an integrable QFT $[43,14]$. Motivated by the fact that also form factors obey $S$-matrix exchange relations they have been interpreted as linear functionals over the $\mathrm{ZF}$ algebra $[34,26]$. Here we shall argue that form factors should more appropriately be regarded as functionals over a larger algebra [28]. In upshot each form factor sequence turns out to be in correspondence to a linear functional over a novel algebra $\mathcal{F}_{\beta}(S)$, which we call "form factor algebra". The relevant functionals are characterized by a simple invariance condition (" $T$-invariance"). From the quantum field theoretical perspective, for $\beta=2 \pi$, each form factor sequence corresponds to a local operator in the QFT aimed at. Symbolically therefore one has the correspondences:

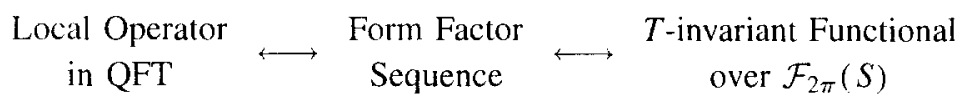

When $\beta$ is different from $2 \pi$ the corresponding sequences or functionals turn out to describe 'Non-Wightman' QFTs with a deformed kinematical arena, still having the same $S$-matrix as the original QFT [29].

The motivation to develop such an algebraic framework is three-fold. First, the formulation avoids making reference to ill-defined traces inevitably showing up otherwise (cf. below). Second, it is amenable to generalizations not visible in the QFT context. Examples are the 'Non-Wightman' QFTs mentioned before, and applications to the quantum Ernst equations. Third, one may hope it to lead to more useful expressions for the form factors in concrete models. So far mostly the solutions of the form factor equations are obtained in the form of multiple contour integrals, where the integrand has complicated monodromy properties $[35,1]$. Since for example a two-point function gets computed from multiple integrals of the modulus square of a form factor, such expressions - though mathematically intriguing in its own right - are not of immediate practical use. Of course it remains to be seen whether the framework here can improve on this.

\subsection{Finite temperature structure}

An important feature of the algebra $\mathcal{F}_{2 \pi}(S)$ is that it unravels the 'finite temperature structure' underlying the form factor approach. The point at issue can be seen from the cyclic form factor equation stating that 


$$
F_{a_{n} \ldots a_{1}}\left(\theta_{n}+2 \pi i, \ldots, \theta_{2}, \theta_{1}\right)=\eta F_{a_{n-1} \ldots a_{1} a_{n}}\left(\theta_{n-1}, \ldots, \theta_{1}, \theta_{n}\right),
$$

where $\eta$ is a phase and the shift by $2 \pi i$ is understood in the sense of analytic continuation. Originally Eq. (1.1) was found in the context of the sine-Gordon model [33] improving on earlier attempts to generalize Watsons equation [20]. Subsequently, Smirnov promoted it to an axiom for the form factors of an integrable QFT, which together with the other equations implies locality [34]. More recently, a derivation of (1.1) from quantum field theoretical principles was given, showing in particular that (1.1) holds in any massive $1+1$ dimensional relativistic QFT, regardless of its integrability [27]. The origin of the phase $\eta$ is clarified in [31].

Evidently Eq. (1.1) has the form of a thermal state, or Kubo-Martin-Schwinger (KMS) condition, with the parameter of the Lorentz boosts playing the role of a 'time' variable. This immediately suggests a relation to the Unruh effect $[6,38,29]$, which however is not straightforward to unravel in the formalism: The Unruh effect proper is strictly limited to free QFTs, in that the Bogoliubov transformation employed in its derivation cannot be constructed otherwise. Its generalization to interacting QFTs is given by the Bisognano-Wichmann theorem [5]. Both lead to KMS conditions for Wightman functions in (complexified) position space. Eq. (1.1) can of course not be obtained by simply Fourier transforming such a position space KMS condition (as sometimes asserted). Rather, Eq. (1.1) is a statement about matrix elements of scattering states, not about Wightman functions. In particular all momenta in (1.1) are on-shell momenta which arise through the asymptotic clustering in a Haag-Ruelle type construction of the scattering states. The thermal properties then have to be rederived from scratch. More details on the derivation of (1.1) can be found in [27].

In the integrable case, after switching to an appropriate pseudo-particle basis, the form factors will in addition obey the familiar $S$-matrix exchange relations. Being a KMS condition (1.1) then superficially suggests to search for solutions of $(1.1)$ in terms of traces over ZF-type operators

$$
F_{a_{1} \ldots a_{1}}\left(\theta_{n}, \ldots, \theta_{1}\right) \stackrel{?}{=} \operatorname{Tr}\left[e^{2 \pi K} \mathcal{O} V_{a_{n}}\left(\theta_{n}\right) \ldots V_{a_{1}}\left(\theta_{1}\right)\right]
$$

where $K$ is the generator of Lorentz boosts, $\mathcal{O}$ represents the local operator, and $V_{u}(\theta)$ are $Z \mathrm{~F}$-type operators satisfying

$$
V_{a}\left(\theta_{1}\right) V_{b}\left(\theta_{2}\right)=S_{a l}^{d c}\left(\theta_{21}\right) V_{c}\left(\theta_{2}\right) V_{d}\left(\theta_{1}\right), \quad \operatorname{Re} \theta_{21} \neq 0, \quad \theta_{21}=\theta_{2}-\theta_{1}
$$

Indeed, such a construction works nicely in the context of lattice models [19] but in a QFT context the relevant trace will never exist and for a very physical reason. Namely $K$ is not like a Hamiltonian, its spectrum is not bounded from below but consists of the entire real axis. In other words, the 'density operator' $e^{2 \pi K}$ is an unbounded operator (and so will be in general also the ZF operators) and the trace is meaningless on any state space on which $K$ has the proper spectrum. Of course one can try to disregard this as a technical nuisance and 'renormalize' the trace in various ways. For example one can divide $(1.2)$ by the equally divergent $\operatorname{Tr}\left[e^{2 \pi K}\right][22,12,6]$. In general, however. there will be no guarantee that the ratio is finite; for example the divergence may 
depend on $\theta_{n}, \ldots, \theta_{1}$ or on the local operator considered. One can also return to a lattice formulation and try to find the proper thermodynamic limit [23], though the trace interpretation of (1.2) is unlikely to survive the limit [17]. Any such procedure however requires a regularization that spoils some of the fundamental features of the QFT aimed at. In addition it is model dependent and against the spirit of the form factor approach, whose most compelling feature is that no regularization and no renormalization is necessary to construct genuine QFT quantities.

In the spirit of the form factor approach one can ask whether it is possible to replace the inevitably sick trace in (1.2) systematically by something well defined. Clearly the required mathematics must be able to deal with thermal states having an unbounded density operator. Fortunately the relevant mathematics is known for almost 30 years and has been found independently in the context of QFT at finite temperature [17] and the structure analysis of von Neumann algebras [40]. The by now common heading is modular structures. It may be helpful to briefly recapitulate their basic features. Modular structures in the context of von Neumann algebras are a pair of operators $(J, \Delta)$ that can be associated to any von Neumann algebra $\mathcal{N}$ with cyclic and separating vector $\Omega$. The latter means that there exists a Hilbert space $\mathcal{H}$ such that both $\mathcal{N} \Omega$ and $\mathcal{N}^{\prime} \Omega$ are dense subspaces of $\mathcal{H}$, where $\mathcal{N}^{\prime}$ is the commutant of $\mathcal{N}$, that is the set (and $C^{*}$ algebra) of all bounded operators on $\mathcal{H}$ commuting with $\mathcal{N}$. The operator $J$ is an antiunitary involution with respect to the inner product on $\mathcal{H}$, and $\Delta$ is a positive self-adjoint (in general unbounded) operator. Further they obey the following defining relations:

$$
\begin{aligned}
& J \Delta^{1 / 2} X \Omega=X^{*} \Omega, \quad \text { for } X \in \mathcal{N}, \\
& J \Delta^{-1 / 2} X^{\prime} \Omega=X^{\prime *} \Omega, \quad \text { for } X^{\prime} \in \mathcal{N}^{\prime} \text {, with } J \Delta J=\Delta^{-1} \text {. }
\end{aligned}
$$

The Tomita-Takesaki theorem [40] states that $J \mathcal{N} J=\mathcal{N}^{\prime}$ and that for all real $\lambda$ the mapping $D_{\lambda}(X)=\Delta^{i \lambda} X \Delta^{-i \lambda}$ defines an automorphism group of both $\mathcal{N}$ and $\mathcal{N}^{\prime}$. In this context we shall refer to the following equation as the "KMS property" of $\Delta$ :

$$
(\Omega, Y \Delta X \Omega)=(\Omega, X Y \Omega), \quad X, Y \in \mathcal{N} .
$$

It follows from the defining relations via $[17,40]$

$$
\begin{aligned}
(\Omega, Y \Delta X \Omega) & =\left(\Delta^{1 / 2} Y^{*} \Omega, \Delta^{1 / 2} X \Omega\right) \\
& =\left(J Y \Omega, J X^{*} \Omega\right)=\left(X^{*} \Omega, Y \Omega\right)=(\Omega, X Y \Omega) .
\end{aligned}
$$

Heuristically one can think of $\Delta$ as being an unbounded density operator for which the relations (1.4), (1.5) provide a substitute for the cyclic property of the trace.

In the context of form factors one is not naturally dealing with von Neumann algebras and the above results do not apply. Nevertheless one can try to develop an algebraic counterpart of this construction, just as the "thermofield formalism" [42] takes the above algebraic relations as the starting point [30], ignoring topological issues in practice. At least for the time being such an algebraic viewpoint seems to be appropriate. Transferred to the form factor situation, a counterpart of the 'modular' or 'thermofield' formalism can be formulated as follows. First one gives up the presupposition that the algebra (1.3) is 
represented irreducibly on the state space. Rather, one works with a manifestly reducible representation. If $W_{a}(\theta)$ denotes the generators of (1.3) in this representation, the reducibility is manifest in that there exists a large class of operators commuting with the $W_{a}(\theta)$ 's, namely a set of operators $j\left(W_{a}\right)(\theta)$ satisfying (1.3) with the complex conjugate $S$-matrix (the "tilde fields" in the thermofield language). Thus

$$
\begin{aligned}
W_{a}\left(\theta_{1}\right) W_{b}\left(\theta_{2}\right) & =S_{a b}^{d c}\left(\theta_{21}\right) W_{c}\left(\theta_{2}\right) W_{d}\left(\theta_{1}\right), \\
j\left(W_{a}\right)\left(\theta_{1}\right) j\left(W_{b}\right)\left(\theta_{2}\right) & =\left[S_{a b}^{d c}\left(\theta_{21}\right)\right]^{*} j\left(W_{c}\right)\left(\theta_{2}\right) j\left(W_{d}\right)\left(\theta_{1}\right), \quad \operatorname{Re} \theta_{21} \neq 0, \\
W_{a}\left(\theta_{1}\right) j\left(W_{b}\right)\left(\theta_{2}\right) & =j\left(W_{b}\right)\left(\theta_{2}\right) W_{a}\left(\theta_{1}\right) .
\end{aligned}
$$

For real boost parameters $\lambda$ the Lorentz boosts act as automorphisms of the algebra (1.7) via $D_{\lambda}\left(W_{a}\right)(\theta)=e^{i \lambda K} W_{a}(\theta) e^{-i \lambda K}=W_{a}(\theta+\lambda)$, etc. For imaginary boost parameters $\lambda$ this is simply meaningless. One of the main results obtained here is as follows: If one starts with an algebra containing a double TTS algebra in addition to an algebra of the form (1.3) and imposes one extra relation, then both $j\left(W_{a}\right)(\theta)$ and $D_{2 \pi i}\left(W_{a}\right)(\theta)$ can be expressed explicitly in terms of $W_{a}(\theta)$ and the generators $T^{ \pm}(\theta)_{a}^{b}$ of the double TTS algebra. Namely

$$
\begin{aligned}
j\left(W_{a}\right)(\theta) & =C_{a a^{\prime}} C^{m n} W_{m}\left(\theta^{*}\right) T^{+}\left(\theta^{*}+i \pi\right)_{n}^{a^{\prime}} \\
& =C_{a a^{\prime}} C^{m n} T^{-}\left(\theta^{*}+i \pi\right)_{n}^{a^{\prime}} W_{m}\left(\theta^{*}+i 2 \pi\right), \\
D_{2 \pi i}\left(W_{a}\right)(\theta) & =C_{m n} T^{-}(\theta+i 2 \pi)_{a}^{m} W_{k}(\theta) T^{+}(\theta+i \pi){ }_{l}^{n} C^{l k}=W_{a}(\theta+i 2 \pi) .
\end{aligned}
$$

Here it is stipulated that $D_{2 \pi i}\left(W_{a}\right)(\theta)=W_{a}(\theta+i 2 \pi)$ is a relation in the new algebra, which we call "modular" algebra $\mathcal{M}_{2 \pi}(S)$, where $S$ refers to the given $S$-matrix and $2 \pi$ is the inverse temperature featuring in the KMS condition. The term "modular" is used because the assignments $j$ and $D_{2 \pi i}$ turn out to be (anti-linear and linear) automorphisms of $\mathcal{M}_{2 \pi}(S)$ having all algebraic properties of modular operators in the context of von Neumann algebras, in particular $j^{2}=\mathrm{id}$. The counterpart of the "thermal ground state" (the cyclic vector in the context of von Neumann algebras) are vectors $\left|\Omega_{2 \pi}\right\rangle$ and $\left\langle\Theta_{2 \pi}\right|$ satisfying

$$
T^{+}(\theta)_{a}^{b}\left|\Omega_{2 \pi}\right\rangle=\delta_{a}^{b}\left|\Omega_{2 \pi}\right\rangle, \quad\left\langle\Theta_{2 \pi}\right| T^{-}(\theta)_{a}^{b}=\eta \delta_{a}^{b}\left\langle\Theta_{2 \pi}\right|,
$$

where in general $\left\langle\Theta_{2 \pi} \mid \Omega_{2 \pi}\right\rangle=0$. One of these vectors, say $\left\langle\Theta_{2 \pi}\right|$, should eventually be thought of as being in correspondence to a local operator in the QFT considered, the other can be viewed as a version of the "Rindler vacuum". The matrix elements

$$
F_{a_{n} \ldots a_{1}}\left(\theta_{n}, \ldots, \theta_{1}\right)=\left\langle\Theta_{2 \pi}\left|W_{a_{n}}\left(\theta_{n}\right) \ldots W_{a_{1}}\left(\theta_{1}\right)\right| \Omega_{2 \pi}\right\rangle
$$

then automatically satisfy (1.1). The usefulness of a "Yangian" or "quantum double" extension of the ZF algebra has been noticed by a number of authors $[9,10,37,4,21,11]$, however without employing the crucial relation (1.8), denoted by (M) (for "modular") below. To simplify the discussion we ignored the residue condition so far. By a suitable modification $\mathcal{F}_{2 \pi}(S)$ of the algebra $\mathcal{M}_{2 \pi}(S)$ one can achieve that the matrix elements (1.7) in fact satisfy all the form factor equations of an integrable QFT without 
bound states. Correspondingly we refer to $\mathcal{F}_{2 \pi}(S)$ as the "form factor algebra". The solution of the recursive system of form factor equations can thus be replaced with the study of the representation theory of $\mathcal{F}_{2 \pi}(S)$.

The rest of the paper is organized as follows. In the next section we define the algebra $\mathcal{F}_{\beta}(S)$, allowing for $\beta$ different from $2 \pi$, and establish the (right half of the) correspondence displayed in Section 1.1. The emergence of the modular structures $(j, \delta)$ is described in Section 3, to be followed by a brief outlook on the perspective.

\section{An algebra underlying the form factor equations}

We work with a slightly generalized set of form factor equations, depending on a real parameter $\beta$. A detailed description is relegated to Appendix A. For $\beta=2 \pi$ they coincide with the form factor equations of an integrable massive QFT without bound states. For generic $\beta$ one obtains a system of deformed form factor equations, whose solutions turn out to define QFTs over some non-commutative space, having the same $S$-matrix as the original QFT [29]. Conceptually the solutions to both systems of equations are sequences of tensor-valued meromorphic functions. Here we show that such sequences can be set into correspondence to linear functionals over an algebra $\mathcal{F}_{\beta}(S)$, which we call "form factor algebra". The relevant functionals are characterized by the " $T$-invariance" condition (1.9).

\subsection{Definition of the algebra}

Let $\mathcal{A}$ denote an abstract normed *-algebra equipped with an $\mathbb{N}$-grading such that the degrees add up upon multiplication of two elements. Let $\mathcal{A}^{(n)}$ be the subspace of degree $n$ and consider the space of mappings $\mathcal{A}^{(m, n)}: D^{m} \rightarrow \mathcal{A}^{(n)}$ continuous on a subset $D^{m}=(\mathbb{R}+I \mathbb{Z})^{m}$ of $\mathbb{C}^{m}$, where $I$ is a finite set of purely imaginary numbers. One expects that such mappings can be generated by 'suitable' multiplication of elements of $\mathcal{A}^{(1.1)}$, i.e. of 1-parameter families $\theta \rightarrow X(\theta)$ of degree-1 operators. However, in general the product of two elements of $\mathcal{A}^{(1.1)}$ will not be continuous on $D^{1} \times D^{1}$. We call

$$
\mathcal{A}(\text { data })=\bigoplus_{m \leqslant n} \mathcal{A}^{(m, n)}
$$

a quantum operator algebra' ${ }^{\prime}$ if the elements of $\mathcal{A}^{(m, n)}$ are generated by two types of product operations from $\mathcal{A}^{(1,1)}$ (and indicate in brackets what kind of data the construction depends on). First, the ordinary product, which will be denoted simply by concatenation of generators. It is assumed to be well defined (at least) whenever $\operatorname{Re} \theta_{1} \neq \operatorname{Re} \theta_{2}$ for $X_{1}\left(\theta_{1}\right), X_{2}\left(\theta_{2}\right) \in \mathcal{A}^{(1,1)}$. By iteration elements of $\mathcal{A}^{(n, n)}$ for arbitrary

\footnotetext{
${ }^{1}$ We borrow the term, though not the concept from Ref. |24|. Apart from the conformal structure the concept bears more resemblance to that in Ref. $|16|$.
} 
$n$ can be generated and will be of the form $X_{1}\left(\theta_{1}\right) \ldots X_{n}\left(\theta_{n}\right)$ with $\operatorname{Re} \theta_{i} \neq \operatorname{Re} \theta_{j}, i \neq$ $j$. Associativity of this product is a consequence of the associativity of the underlying algebra $\mathcal{A}$. Second we assume that there exists a contraction product, which is defined whenever a difference $\pm\left(\theta_{1}-\theta_{2}\right)$ assumes one out of the finite number of purely imaginary values $l$. The contraction product is again defined recursively starting from

$$
\text { . }: \mathcal{A}^{(1,1)} \times \mathcal{A}^{(1,1)} \longrightarrow \mathcal{A}^{(0,1)} .
$$

The extension to other $\mathcal{A}^{(m, n)}$ is done by assuming compatibility with the ordinary product $X \cdot(Y Z)=(X \cdot Y) Z$. We shall not assume that the contraction product is associative by itself, though it may turn out to be in specific cases. Clearly a quantum operator algebra can be specified in terms of the generators $\mathcal{A}^{(1,1)}$ and $\mathcal{A}^{(0,1)}$, the set $I$, and the two products. Note that elements of infinite degree are not defined. A quantum operator algebra will be called Lorentz covariant if translations in the variable $\operatorname{Re} \theta$ are unitarily implemented; the generator of the automorphism group will be denoted by $K$. Explicitly on the degree- 1 elements this means $e^{i \lambda K} X(\theta) e^{-i \lambda K}=X(\theta+\lambda), \lambda \in \mathbb{R}$.

In the following we define four Lorenty covariant quantum operator algebras associated with a given bootstrap $S$-matrix. In addition they depend on a real parameter $\beta$ and the index set $I=\{i \pi, i(\beta-\pi)\}$. The charge conjugation matrix and its inverse are identified with central elements $C_{a b}, C^{a b} \in \mathcal{A}^{(0,0)} \subset \mathcal{A}^{(1,0)}$, where the inclusion treats $C_{a b}, C^{a b}$ as constant functions in $\mathcal{A}^{(1.0)}$. We shall write $\mathcal{A}_{\beta}(S)$ for $\mathcal{A}(S, I, \beta)$ in $(2.1)$ and use different symbols for the various algebras, but keep the generic notation $\mathcal{A}^{(m, n)}$ for their grade spaces. The algebras are

(1) A generalized quantum double $\mathcal{T}_{\beta}(S)$ with generators $T^{ \pm}(\theta)_{a}^{b}$.

(2) The modular algebra $\mathcal{M}_{\beta}(S)$ with generators $T^{ \pm}(\theta)_{a}^{b}$ and $W_{a}(\theta)$.

(3) An auxiliary algebra $\mathcal{F}_{\beta}^{ \pm}(S)$ with generators $T^{ \pm}(\theta)_{a}^{b}$ and $W_{a}^{ \pm}(\theta)$.

(4) The form factor algebra $\mathcal{F}_{\beta}(S)$ being the subalgebra of $\mathcal{F}_{\beta}^{ \pm}(S)$ generated by $T^{ \pm}(\theta)_{a}^{b}$ and $W_{a}(\theta)=W_{a}^{+}(\theta)+W_{a}^{-}(\theta)$.

The indices $a, b$, etc., refer to the modules $V_{a}, V_{b}$ associated with the given bootstrap $S$ matrix $S$, cf. Appendix $A$. The grading is such that $W_{a}(\theta), W_{a}(\theta)^{ \pm}, T^{ \pm}(\theta)_{a}^{b} \in \mathcal{A}^{(1,1)}$. The two products will be defined by specifying relations among the generators.

Definition of $\mathcal{T}_{\beta}(S)$ and $\mathcal{M}_{\beta}(S)$

The defining relations of $\mathcal{T}_{\beta}(S)$ are

$$
\begin{aligned}
& S_{m m}^{c d}\left(\theta_{12}\right) T^{ \pm}\left(\theta_{1}\right)_{a l}^{n} T^{ \pm}\left(\theta_{2}\right)_{b}^{m}=T^{ \pm}\left(\theta_{2}\right)_{n}^{c} T^{ \pm}\left(\theta_{1}\right)_{m}^{d} S_{a b}^{m m}\left(\theta_{12}\right), \\
& S_{m m}^{c d}\left(\theta_{12}\right) T^{+}\left(\theta_{1}\right)_{a}^{n} T^{-}\left(\theta_{2}\right)_{b}^{m}=T^{-}\left(\theta_{2}\right)_{n}^{c} T^{+}\left(\theta_{1}\right)_{m}^{d d} S_{a b}^{m n}\left(\theta_{12}+i 2 \pi-i \beta\right),
\end{aligned}
$$

valid for $\operatorname{Re} \theta_{12} \neq 0, \theta_{12}:=\theta_{1}-\theta_{2}$. Further

$$
\begin{aligned}
& C_{m n} T^{ \pm}(\theta)_{a}^{m} \cdot T^{ \pm}(\theta-i \pi)_{b}^{n}=C_{a b}, \\
& C^{m n} T^{ \pm}(\theta)_{m}^{a} \cdot T^{ \pm}(\theta+i \pi)_{n}^{b}=C^{a b} . \\
& C_{m n} T^{+}(\theta)_{a}^{m} \cdot T^{+}(\theta+i \pi)_{b}^{n}=C_{m ! n} T^{-}(\theta-i \beta+i 2 \pi)_{a}^{m} \cdot T^{-}(\theta-i \beta+i 3 \pi)_{b}^{n}, \\
& C^{m n} T^{+}(\theta)_{m}^{a} \cdot T^{+}(\theta-i \pi)_{n}^{b}=C^{m m} T^{-}(\theta)_{m}^{a} \cdot T^{-}(\theta-i \pi)_{n}^{n} .
\end{aligned}
$$


The ', ' product in $\mathcal{T}_{\beta}(S)$ is associative. For later use notice that there are two degree-0 elements in $\mathcal{T}_{\beta}(S)$ that do not appear in the defining relations, namely

$$
\begin{aligned}
& C_{a b}(\theta):=C_{m n} T^{+}(\theta)_{a}^{m} \cdot T^{+}(\theta+i \pi)_{b}^{n} \in \mathcal{A}^{(1,0)} \\
& C^{a b}(\theta):=C^{m n} T^{+}(\theta)_{m}^{a} \cdot T^{+}(\theta-i \pi)_{n}^{b} \in \mathcal{A}^{(1,0)},
\end{aligned}
$$

where (T3) can be used to obtain equivalent expressions in terms of $T^{-}$.

Now extend the algebra $\mathcal{T}_{\beta}(S)$ to $\mathcal{M}_{\beta}(S)$ by adding generators $W_{a}(\theta)$ having the following linear exchange relations with $T^{ \pm}(\theta)_{a}^{b}$ :

$$
\begin{aligned}
& T^{-}\left(\theta_{1}\right)_{a}^{e} W_{b}\left(\theta_{2}\right)=S_{a b}^{d c}\left(\theta_{12}\right) W_{c}\left(\theta_{2}\right) T^{-}\left(\theta_{1}\right)_{d}^{e}, \\
& T^{+}\left(\theta_{1}\right)_{a}^{e} W_{b}\left(\theta_{2}\right)=S_{a b}^{d c}\left(\theta_{12}+i 2 \pi-i \beta\right) W_{c}\left(\theta_{2}\right) T^{+}\left(\theta_{1}\right)_{d}^{e} .
\end{aligned}
$$

These relations hold for all relative rapidities, including $\operatorname{Re} \theta_{12}=0$. Further impose

$$
\begin{aligned}
& W_{a}\left(\theta_{1}\right) W_{b}\left(\theta_{2}\right)=S_{a b}^{d c}\left(\theta_{12}\right) W_{c}\left(\theta_{2}\right) W_{d}\left(\theta_{1}\right), \quad \operatorname{Re} \theta_{12} \neq 0 . \\
& C^{m m} W_{m}(\theta) \cdot T^{+}(\theta+i \beta-i \pi)_{n}^{a}=C^{m n} T^{-}(\theta+i \beta-i \pi)_{n}^{a} \cdot W_{m}(\theta+i \beta) .
\end{aligned}
$$

The relation ( $\mathrm{M}$ ) will later turn out to implement the action of the modular operators on $\mathcal{M}_{\beta}(S)$. The ' $\because$ product on $\mathcal{M}_{\beta}(S)$ is associative. An equivalent form of $(\mathrm{M})$ is

$$
\begin{aligned}
& W_{a}(\theta+i \beta)=C_{m n} T^{-}(\theta+i \beta)_{a}^{m} \cdot W_{k}(\theta) \cdot T^{+}(\theta+i \beta-i \pi)_{l}^{n} C^{k l} \\
& W_{a}(\theta-i \beta)=C^{k l} T^{-}(\theta-i \pi)_{l}^{\prime} \cdot W_{k}(\theta) \cdot T^{+}(\theta-i 2 \pi)_{a}^{m} C_{m !}
\end{aligned}
$$

\section{Definition of $\mathcal{F}_{\beta}^{ \pm}(S)$ and $\mathcal{F}_{\beta}(S)$}

Now take two copies of a $\mathcal{M}_{\beta}(S)$ algebra, which share the same $T^{ \pm}$generators but have distinct $W$-generators $W_{a}^{+}(\theta)$ and $W_{a}^{-}(\theta)$. Among them the following relations hold: ${ }^{2}$

$$
\begin{aligned}
& W_{a}^{-}(\theta+i \pi) \cdot W_{b}^{-}(\theta)=-\lambda^{-} C_{a b}, \\
& W_{a}^{+}(\theta-i \pi) \cdot W_{b}^{+}(\theta)=-\lambda^{+} C_{a b}(\theta+i \beta-i 3 \pi),
\end{aligned}
$$

for constants $\lambda^{ \pm} \in \mathbb{C}$. All other contraction products $W_{a}^{ \pm}\left(\theta_{1}\right) \cdot W_{b}^{ \pm}\left(\theta_{2}\right)$ and $W_{a}^{ \pm}\left(\theta_{1}\right)$. $W_{b}^{\mp}\left(\theta_{2}\right), \pm \theta_{12} \in I$ vanish. Finally impose

$$
W_{a}^{+}\left(\theta_{1}\right) W_{b}^{-}\left(\theta_{2}\right)=S_{a b}^{d c}\left(\theta_{12}\right) W_{c}^{-}\left(\theta_{2}\right) W_{d}^{+}\left(\theta_{1}\right), \quad \operatorname{Re} \theta_{12} \neq 0 .
$$

$(\mathrm{W} \pm)$

This concludes the definition of the algebra $\mathcal{F}_{\beta}^{ \pm}(S)$. The form factor algebra $\mathcal{F}_{\beta}(S)$ is the algebra generated by $T^{ \pm}(\theta)$ and the sum $W_{a}(\theta)=W_{a}^{+}(\theta)+W_{a}^{-}(\theta)$, subject to the relations induced from $\mathcal{F}_{\beta}^{ \pm}(S)$. It is easy to see that only the relations ( $\mathrm{R} \pm$ ) change in a way detailed in Section 2.2 .

\footnotetext{
${ }^{2}$ Here $C_{a b}(\theta)$ can be replaced with $C_{a b}$ without affecting the consistency of the algebra or any other of the results of Section 2 . When the $S$-matrix is $2 \pi i$-periodic, this replacement becomes an identity; cf. Section 2.2 .
} 
Implicit in these definitions, of course, is the presupposition that the above relations define consistent algebras.

Proposition I. The algebras (1)-(4) are consistent (in the sense of the proof). The ordinary product is associative and compatible with the contraction product.

Proof. (Sketch) One has to show that the defining relations for both the ordinary product and the contraction product arise from dividing out two-sided ideals in the respective 'free' algebras, where no relations among the generators are imposed. Once this is known, associativity of the ordinary product follows from the assumed associativity of the underlying *-algebra. Consistency with the contraction product is assumed when checking the ideals and then justified in retrospect. The search for ideals and the construction of the successive quotient algebras is best done in a particular order. The principle is largely analogous to that in [28], so that it may be sufficient to list the items to be checked and to make a few comments on each entry.

(a) Consistency and associativity of $\mathcal{T}_{\beta}(S)$ : Because of (T3) and the $\beta$-dependence this does not follow from the quantum double construction. Concerning the relations ( 1 1) there are in principle six consistency conditions to be checked, which arise from pushing $T^{+}$or $T^{-}$through one of the relations (T1). Using the known consistency of the $\beta$-independent equations and the homomorphism (2.11) below, only two of them have to be checked explicitly. Doing this one establishes the consistency and, as a byproduct, the associativity of the algebra with relations (T1). In this algebra the relations (T2) and (T3) are found to correspond to two-sided ideals. In checking this for (T3) relations like

$$
S_{c a}^{m n n}(\theta+i \pi) S_{m b}^{k \prime}(\theta) C_{m l}=C_{a b} \delta_{c}^{k}
$$

are useful. The latter can be verified by inserting $C_{n l}=-\operatorname{dim} V S_{n l}^{n q}(-i \pi) C_{p q}$ (which is regular even if $S_{m l}^{\prime q}(-i \pi)$ is singular) and use of the Yang Baxter equation. Dividing out these ideals one establishes the consistency of $\mathcal{T}_{\beta}(S)$ and its associativity with respect to both products.

(b) Consistency of (TW) and (WW) with $\mathcal{T}_{\beta}(S)$ : First one checks the consistency of (TW) with $\mathcal{T}_{\beta}(S)$ by pushing $T^{ \pm}$through (TW) and $W$ through (T1), (T2), (T3). Similarly one verifies the consistency of (TW) and (WW). Finally one shows that the relations (T1), (T2), (T3) arise from two-sided ideals in the associative algebra with relations (TW) and (WW) only.

(c) Consistency of (M) with all other relations: One can verify that (M) arises from a two-sided ideal in the algebra with relations (TW), (WW), (T1). This fact holds for any relative coefficient between the left and the right-hand side of (M). In particular the phase $\eta$ appearing in (1.9) could also be incorporated here. It is however more natural to attribute $\eta$ to the state rather than the algebra. Dividing out the ideal corresponding to (M) the consistency of the algebra $\mathcal{M}_{\beta}(S)$ follows.

(d) Consistency of $(\mathrm{R} \pm$ ) with all other relations: The point to verify here is that assignments of the form $(\mathrm{R}-): W_{l l}(\theta+i \pi) \cdot W_{b}(\theta)=-\lambda^{-} C_{a l}$ and $(\mathrm{R}+)$ : 
$W_{a}(\theta-i \pi) \cdot W_{b}(\theta)=-\lambda^{+} C_{a b}(\theta+i \beta-i 3 \pi)$ are algebraically consistent. For that purpose one can drop the notational distinction between $W^{+}$and $W^{-}$. Again the differences of both sides of the relations $(\mathrm{R} \pm$ ) have to generate two-sided ideals in the algebra without the $(\mathrm{R} \pm)$ relations imposed. For $(\mathrm{R}-)$ this is a trivial consequence of the crossing relation of the $S$-matrix. For $(\mathrm{R}+)$ it is a consequence of the following exchange relations between $C_{a b}(\theta)$ and $W_{a}(\theta), T^{ \pm}(\theta)_{a i}^{b}$ :

$$
\begin{aligned}
S_{a b}^{m n}\left(\theta_{21}+i \beta-i 3 \pi\right) C_{c n}\left(\theta_{1}\right) W_{m}\left(\theta_{2}\right) & =S_{a c}^{m n}\left(\theta_{12}-i \beta+i 2 \pi\right) W_{m}\left(\theta_{2}\right) C_{n b}\left(\theta_{1}\right), \\
S_{a b}^{m n}\left(\theta_{21}+i \beta-i 3 \pi\right) C_{c n}\left(\theta_{1}\right) T^{-}\left(\theta_{2}\right)_{m}^{d} & =S_{a c}^{m n}\left(\theta_{12}-i \beta+i 2 \pi\right) T^{-}\left(\theta_{2}\right)_{m}^{d} C_{m b}\left(\theta_{1}\right), \\
S_{a b}^{m n}\left(\theta_{21}-i \pi\right) C_{c n}\left(\theta_{1}\right) T^{+}\left(\theta_{2}\right)_{m}^{d} & =S_{a c}^{m n}\left(\theta_{12}\right) T^{+}\left(\theta_{2}\right)_{m}^{d} C_{n b}\left(\theta_{1}\right) .
\end{aligned}
$$

As remarked in footnote 2 the assignment $(R+)$ is also consistent with the reduction $C_{a b}(\theta) \rightarrow C_{a b}$. The reduced equation $(R+)$ is found to be consistent by means of (2.5). In particular this shows that assignments of the form $W_{a}(\theta+i \alpha) \cdot W_{b}(\theta)=$ (Central element $)_{a b}$ with some parameter $\alpha \neq \pm i \pi$ are inconsistent.

This concludes the verification of the consistency of the algebras (1)-(4). Next we discuss some of their basic properties.

\subsection{Basic properties}

Consider first the algebra $\mathcal{T}_{\beta}(S)$ in more detail. From the definitions (2.3) and (T2) one finds

$$
\begin{aligned}
T^{+}(\theta)_{m}^{b} \cdot C^{m n} C_{m a}(\theta+i \pi) & =T^{+}(\theta+i 2 \pi)_{a}^{b}, \\
T^{-}(\theta)_{m}^{b} \cdot C^{m m} C_{n a}(\theta+i \beta-i \pi) & =T^{-}(\theta+i 2 \pi)_{a}^{b}, \\
T^{ \pm}(\theta)_{a}^{m} \cdot C_{m n} C^{n b}(\theta-i \pi) & =T^{ \pm}(\theta-i 2 \pi)_{a}^{b}
\end{aligned}
$$

Therefore, shifts by $2 \pi i$ in the arguments can be implemented within $\mathcal{T}_{\beta}(S)$. When the $S$-matrix is $2 \pi i$-periodic we show in the next paragraph that $C_{a b}(\theta)$ and $C^{a b}(\theta)$ reduce to $C_{a b}$ and $C^{a b}$, respectively. By (2.7) then also $T^{ \pm}(\theta)$ are $2 \pi i$-periodic, consistent with (T1).

The relation (T3) is needed to guarantee that the operator $C_{a b}(\theta)$, although not central, behaves as $C_{a b}$ in certain functionals (cf. Eq. (2.30) below) and similarly for $C^{a b}(\theta)$. The latter feature reflects the fact that the condition $(\mathrm{R}+)$ could be replaced with $(\mathrm{R}+)^{\prime}: W_{a}^{+}(\theta-i \pi) \cdot W_{b}^{+}(\theta)=-\lambda^{+} C_{a b}$, without affecting the consistency of the algebra $\mathcal{F}_{\beta}(S)$ or the validity of Theorem 1 below. The full form of $(\mathrm{R}+)$ as given before enters however crucially in Section 3, at least when the $S$-matrix is not $2 \pi i$-periodic. When the $S$-matrix is $2 \pi i$-periodic - and only then - the following simplifications take place: (i) The operators $C_{a b}(\theta)$ and $C^{a b}(\theta)$ reduce to $C_{a b}$ and $C^{a b}$, respectively. In particular (T3) becomes a trivial consequence of (T2). (ii) The operators $T^{ \pm}(\theta)_{a}^{b}$ are $2 \pi i$-periodic. (iii) The relation ( $\mathrm{T} 1$ ) can be assumed to hold also for $\operatorname{Re} \theta_{12}=0$.

To see point (i) note the relations 


$$
\begin{aligned}
& S_{a b}^{m n}(i \pi) C_{m n}(\theta)=-\operatorname{dim} V C_{a b}, \\
& S_{m n}^{a b}(i \pi) C^{m n}(\theta)=-\operatorname{dim} V C^{a b} .
\end{aligned}
$$

Thus, provided $S_{a b}^{d c}(-i \pi)$ is well defined, - which holds in particular when the $S$-matrix is $2 \pi i$-periodic - one deduces upon contraction

$$
C_{a b}(\theta)=C_{a b}, \quad C^{a b}(\theta)=C^{a b} .
$$

As a by-product one finds that the regularity of $S_{a b}^{d c}(-i \pi)$ is also a sufficient condition for the $S$-matrix to be $2 \pi i$-periodic, provided the relations (T1), (T2) hold (for $\operatorname{Re} \theta_{12} \neq$ $0)$. Indeed, if $S_{d b}^{d c}(-i \pi)$ is regular one concludes from $(2.7)$, (2.9) that the operators $T^{ \pm}(\theta)_{a l}^{b}$ are $2 \pi i$-periodic. Since the relations ( $\mathrm{Tl}$ ) with periodic $T^{ \pm}$operators only make sense if also the $S$-matrix is periodic, one obtains the implication

$(\mathrm{T} 1),(\mathrm{T} 2): \quad S_{a b}^{d c}(-i \pi)$ is regular $\Longrightarrow S_{a b}^{d c}(\theta)$ is $2 \pi i$-periodic.

This in turn implies the dichotomy announced in (A.5). The point with (iii) is that assuming ( $\mathrm{Tl}$ ) to hold also for $\operatorname{Re} \theta_{12}=0$ the $2 \pi i$-periodicity of $T^{ \pm}$can be deduced from (T2). Thus, the former only makes sense if the latter holds anyway, i.e. if the $S$-matrix is $2 \pi i$-periodic. To see the implication (iii) $\Rightarrow$ (ii) it suffices to show that (iii) implies (2.9). Consider first $C_{a b}(\theta)=C_{n ! n} T^{+}(\theta)_{a}^{m} \cdot T^{+}(\theta+i \pi)_{b}^{n}$. Inserting $C_{m m}=$ $-\operatorname{dim} V S_{m m}^{q q}(-i \pi) C_{p q}$ and using (T1) for $\theta_{1}=\theta, \theta_{2}=\theta+i \pi$, a further application of (T2) reduces the expression to $C_{\text {air }}$. The second relation (2.9) is obtained similarly.

Apart from the relation (T3) the algebra $\mathcal{T}_{\beta}(S)$ is a well-known structure. For $\beta=2 \pi$ it can be viewed as a presentation of the quantum double of some underlying infinite dimensional Hopf algebra. The (TW) relations are then characteristic for the intertwining operators between quantum double modules [15,4,21]. Particular cases are the Yangian double or the quantum double of $U_{q}(\hat{g})$ in which case the parameter $\beta$ can be related to the central extension via $\hbar c=i(2 \pi-\beta)[18]$. Here we do not make use of the co-algebra structure and always treat $\beta$ as a (real) numerical parameter entering the algebra via the set $I$ and the relations (M).

Concerning the role of this parameter $\beta$ first notice that $T^{+}$and $T^{-}$enter asymmetrically in $\mathcal{T}_{\beta}(S)$; the flip isomorphism is given by

$$
\mathcal{T}_{\beta}(S) \longrightarrow \mathcal{T}_{4 \pi-\beta}(S), \quad T^{ \pm}(\theta)_{a}^{b} \longrightarrow T^{\mp}(\theta+i \beta-i 2 \pi)_{a}^{b} .
$$

Further, (T1) has the usual consequences for the traces $t^{ \pm}(\theta)=T^{ \pm}(\theta)_{a}^{a}$ separately, i.e.

$$
\left[t^{ \pm}\left(\theta_{1}\right), t^{ \pm}\left(\theta_{2}\right)\right]=0
$$

but $t^{+}\left(\theta_{1}\right)$ and $t^{-}\left(\theta_{1}\right)$ will no longer commute for $\beta \neq 2 \pi$. The value of $\beta$ in the full algebra $\mathcal{M}_{\beta}(S)$ is in fact fixed only by the relation (M). Dropping the relation (M) the algebras with (generic) parameter values $\beta_{1}$ and $\beta_{2}$ would be isomorphic. The (up to overall shifts of the rapidity on the r.h.s.) unique isomorphism is given by

$$
\begin{aligned}
W_{a}(\theta) & \longrightarrow W_{a}(\theta), \\
T^{+}(\theta)_{a}^{b} & \longrightarrow T^{+}\left(\theta+i \beta_{2}-i \beta_{1}\right)_{a}^{b}, \quad T^{-}(\theta)_{a}^{b} \longrightarrow T^{-}(\theta)_{a}^{b} .
\end{aligned}
$$


Clearly this fails to provide a homomorphism for the relation (M) and the algebras $\mathcal{M}_{\beta_{1}}(S)$ and $\mathcal{M}_{\beta_{2}}(S)$ for $\beta_{1} \neq \beta_{2}$ cannot be related by the shift isomorphism $(2.12)$. A fortiori this holds for $\mathcal{F}_{\beta}(S)$.

The relation $(\mathrm{M})$ also allows one to compute the contraction product of $W$ generators at all relative rapidities $\theta_{12}=i \pi+i p \beta$ and $\theta_{12}=-i \pi+i p \beta, p \in \mathbb{Z}$. In particular one finds that the contraction product of two $W^{-}$generators vanishes at $\theta_{12}=i \pi+i p \beta$ and is non-zero at $\theta_{12}=-i \pi+i p \beta$. Similarly the contraction product of two $W^{+}$ generators vanishes at $\theta_{12}=-i \pi+i p \beta$ and is non-trivial at $\theta_{12}=i \pi+i p \beta$. For $p=1$ the contractions are

$$
\begin{aligned}
& W_{a}^{+}(\theta+i \beta-i \pi) \cdot W_{b}^{+}(\theta)=\lambda^{+} \operatorname{dim} V D_{a b}^{+}(\theta+i \beta-2 i \pi), \\
& W_{a}^{-}(\theta-i \beta+i \pi) \cdot W_{b}^{-}(\theta)=\frac{1}{\operatorname{dim} V} \lambda^{-} D_{a b}^{-}(\theta-i \pi),
\end{aligned}
$$

where

$$
\begin{aligned}
& D_{a b}^{+}(\theta):=C_{m n} T^{-}(\theta+i \pi)_{a}^{m} \cdot T^{+}(\theta)_{b}^{n}, \\
& D_{a b}^{-}(\theta):=-\operatorname{dim} V S_{a b}^{d c}(i \pi-i \beta) D_{c d}^{+}(\theta) .
\end{aligned}
$$

When the $S$-matrix is $2 \pi i$-periodic $D_{a b}^{-}(\theta)$ is defined for all real $\beta$. Further since then (T1) is also valid for $\operatorname{Re} \theta_{12}=0$ an alternative expression for $D_{a b}^{-}(\theta)$ is

$$
D_{a b}^{-}(\theta)=C_{m n} T^{+}(\theta)_{a}^{m} \cdot T^{-}(\theta+i \pi)_{b}^{n} .
$$

In the non-periodic case $\beta=2 \pi$ must be excluded. For the sum $W_{a}(\theta)=W_{a}^{+}(\theta)+$ $W_{a}^{-}(\theta)$ the relations (2.13) imply

$\beta$ generic: $\quad W_{a}(\theta+i \pi) \cdot W_{b}(\theta)=-\lambda^{-} C_{a b}$,

$$
\begin{array}{cl} 
& W_{a}(\theta-i \pi) \cdot W_{b}(\theta)=-\lambda^{+} C_{a b}(\theta+i \beta-i 3 \pi), \\
& W_{a}(\theta+i \beta-i \pi) \cdot W_{b}(\theta)=\lambda^{+} \operatorname{dim} V D_{a b}^{+}(\theta+i \beta-2 i \pi), \\
& W_{a}(\theta-i \beta+i \pi) \cdot W_{b}(\theta)=\frac{1}{\operatorname{dim} V} \lambda^{-} D_{a b}^{-}(\theta-i \pi), \\
\beta=2 \pi: \quad & W_{a}(\theta+i \pi) \cdot W_{b}(\theta)=\lambda^{+} \operatorname{dim} V D_{a b}^{+}(\theta)-\lambda^{-} C_{a b}, \\
& W_{a}(\theta-i \pi) \cdot W_{b}(\theta)=\frac{1}{\operatorname{dim} V} \lambda^{-} D_{a b}^{-}(\theta-i \pi)-\lambda^{+} C_{a b}(\theta-i \pi) .
\end{array}
$$

For the $W^{ \pm}$generators the (WW) and $(\mathrm{W} \pm)$ relations break down at purely imaginary relative rapidities. For their sum $W_{a}(\theta)=W_{a}^{+}(\theta)+W_{a}^{-}(\theta)$ the contraction products $(2.16),(2.17)$ are consistent with an extension of the exchange relations (WW) to purely imaginary relative rapidities

$$
\begin{gathered}
W_{a}(\theta+i \pi) \cdot W_{b}(\theta)=-S_{a b}^{d c}(i \pi) W_{c}(\theta) \cdot W_{d}(\theta+i \pi), \\
W_{a}(\theta+i \beta-i \pi) \cdot W_{b}(\theta)=-S_{a b}^{d c}(i \beta-i \pi) W_{c}(\theta) \cdot W_{d}(\theta+i \beta-i \pi),
\end{gathered}
$$


provided $\lambda^{+}$and $\lambda^{-}$are related by

$$
\lambda^{+}=\frac{\lambda^{-}}{\operatorname{dim} V}
$$

Anticipating the later interpretation of the WW contraction product as a residue (within certain functionals), Eqs. (2.18) are what one would expect if the exchange relations (WW) were valid also at purely imaginary relative rapidities. Henceforth we shall take $\lambda^{ \pm}$to be purely imaginary $\lambda^{-}=-2 i, \lambda^{+}=-2 i / \operatorname{dim} V$.

Finally we note that the algebras $\mathcal{T}_{\beta}(S), \mathcal{M}_{\beta}(S)$ and $\mathcal{F}_{\beta}(S)$ are equipped with a *-operation. In technical terms a *-operation is an antilinear anti-involution of some associative algebra. Here we denote such operations by $\sigma$ since $*$ is already used for complex conjugation and $\dagger$ would be cumbersome. The algebras $\mathcal{T}_{\beta}(S), \mathcal{M}_{\beta}(S)$ and $\mathcal{F}_{\beta}(S)$ admit an antilinear anti-involution $\sigma$ given by

$$
\sigma T^{ \pm}(\theta)_{a}^{b}=T^{\mp}\left(\theta^{*}+i \beta-i \pi\right)_{a}^{b}, \quad \sigma W_{a}(\theta)=W_{a}\left(\theta^{*}+i \pi\right), \quad \sigma^{2}=\mathrm{id} .
$$

It is unique up to overall shifts of $\theta$ on the right-hand side by a purely imaginary number; the choice (2.20) adheres to the crossing relations for the form factor equations.

\subsection{T-invariant functionals and form factor sequences}

Consider now linear functionals $\omega_{\beta}: \mathcal{F}_{\beta}(S) \rightarrow \mathbb{C}$. We call a linear functional $T$ invariant if it Lorentz invariant $\omega_{\beta}\left(e^{i \lambda K} X\right)=\omega_{\beta}\left(X e^{i \lambda K}\right)=\omega_{\beta}(X), \lambda \in \mathbb{R}$ and satisfies

$$
\omega_{\beta}\left(T^{-}(\theta)_{a}^{b} X\right)=\eta \delta_{a}^{b}, \quad \omega_{\beta}\left(X T^{+}(\theta)_{a}^{b}\right)=\delta_{a}^{b},
$$

for all elements $X \in \mathcal{F}_{\beta}(S)$ with rapidities separated from $\theta$. An element $X \in \mathcal{A}^{(n, n)}$ depending on rapidities $\theta_{1}, \ldots, \theta_{m}$ is said to have rapidities separated from $\theta$, if $\theta_{j}-\theta \notin$ $\mathbb{Z} I, 1 \leqslant j \leqslant m$. The functionals (2.21) are the form factor analogue of the thermal equilibrium states and will turn out to be invariant under the (counterpart of the) action of the modular operators. Important examples of such functionals are vector functionals (not traces!)

$$
\omega_{\beta}(X)=\left\langle\Theta_{\beta}|X| \Omega_{\beta}\right\rangle
$$

built from a pair of vectors $\left|\Omega_{\beta}\right\rangle$ and $\left\langle\Theta_{\beta}\right|$ satisfying

$$
T^{+}(\theta)_{a}^{b}\left|\Omega_{\beta}\right\rangle=\delta_{a}^{b}, \quad\left\langle\Theta_{\beta}\right| T^{-}(\theta)_{a}^{b}=\eta \delta_{a}^{b},
$$

where in general $\left\langle\Theta_{\beta} \mid \Omega_{\beta}\right\rangle=0$. We shall later address the question as to what extent these vector functionals are generic. Here observe that any $T$-invariant functional $(2.21)$ is uniquely determined by its values on strings of $W$-generators, for which we introduce some extra notation

$$
f_{a_{n} \ldots a_{3}}\left(\theta_{n}, \ldots, \theta_{1}\right):=\omega_{\beta}\left(W_{a_{n}}\left(\theta_{n}\right) \ldots W_{a_{1}}\left(\theta_{1}\right)\right),
$$


where $\operatorname{Re} \theta_{i j} \neq 0, i \neq j$. Sometimes also the shorthand $f^{(n)}$ for the value of $\omega_{\beta}$ on a string of $n W$-generators will be used. We can now partially restore topological concepts by calling a $T$-invariant functional analytic if (i) the dependence of the values $f^{(n)}$ on the parameters $\theta_{1}, \ldots, \theta_{n}$ is locally analytic; (ii) overall shifts $\theta_{j} \rightarrow \theta_{j}+i \beta / 2$ leave the values $f^{(n)}$ invariant up to possibly a phase; (iii) $\omega_{\beta}\left(X W_{a}\left(\theta_{1}\right) W_{b}\left(\theta_{2}\right) Y\right.$ ) has simple poles at $\pm \theta_{12} \in I$ whose residues are given by $\omega_{\beta}\left(X W_{a}\left(\theta_{1}\right) \cdot W_{b}\left(\theta_{2}\right) Y\right), \pm \theta_{12} \in I$, for elements $X, Y$ with rapidities separated from $\theta_{1}, \theta_{2}$. Further, a $T$-invariant functional $\omega_{\beta}$ is called hermitian if

$$
f_{a_{1} \ldots a_{n}}\left(\theta_{1}^{*}+i \pi, \ldots, \theta_{n}^{*}+i \pi\right)=f_{a_{n} \ldots a_{1}}\left(\theta_{n}, \ldots, \theta_{1}\right)^{*} .
$$

For generic elements $X \in \mathcal{A}^{(m, n)}$ this amounts to

$$
\omega_{\beta}(\sigma(X))=\eta^{\prime} \omega_{\beta}(X)^{*},
$$

for some $X$-dependent integer $l$, which can be computed from (2.21) and (2.25). A $T$-invariant analytic and hermitian linear functional over $\mathcal{F}_{\beta}(S)$ will be called a $T$ invariant form. Using the definition (2.21), and the relations of the algebra $\mathcal{F}_{\beta}(S)$ one can write down a system of functional equations for the matrix elements (2.24) whose consistency is guaranteed by that of the underlying algebra $\mathcal{F}_{\beta}(S)$.

Theorem 1. For any $T$-invariant form $\omega_{\beta}$ the sequences $\left(f^{(n)}\right)_{n \geqslant 1}$ satisfy the coupled system (I), (II) of functional equations described in Appendix A.

The proof is a direct application of the defining relations together with (2.16), (2.17) [28]. The consistency of the resulting functional equations is ensured by the consistency of the underlying algebra. In particular any $T$-invariant functional over the modular algebra $\mathcal{M}_{\beta}(S)$ produces solutions of the equivariance equations (I) for all $n \geqslant 1$. For $\beta=2 \pi$ Eqs. (I), (II) coincide with the form factor equations of a massive integrable QFT without bound states. For $\beta \neq 2 \pi$ one gets a modified system of equations, and its solutions no longer describe the form factors of a standard QFT. The elements of a sequence $\left(f^{(n)}\right)_{n \geqslant 1}$ will be called "form factors" for $\beta=2 \pi$ and "deformed form factors" for $\beta \neq 2 \pi$, or, when the distinction is inessential, simply "form factors". The theorem then implies that each $T$-invariant form over $\mathcal{F}_{\beta}(S)$ is uniquely determined by a sequence of form factors and vice versa, i.e. one has a one-to-one correspondence

$$
\omega_{\beta} \longleftrightarrow\left(f^{(1)}, f^{(2)}, \ldots, f^{(n)}, \ldots\right) .
$$

Comparing (2.25) with (1.1) one sees that hermitian linear forms correspond to form factors of hermitian operators. Theorem 1 also holds without this restriction, but for the moment we impose (2.26) for convenience.

To each $T$-invariant form $\omega_{\beta}$ a canonical quadratic form $(,)_{\omega}: \mathcal{F}_{\beta}(S) \times \mathcal{F}_{\beta}(S) \rightarrow \mathbb{R}$ can be associated such that 'off the diagonals', that is, whenever all rapidities of $X$ are separated from all of $Y$, it obeys

$$
(Y, X)_{\omega}=\omega_{\beta}(\sigma(Y) X) .
$$


The explicit expression, valid also 'on the diagonals', is relegated to Appendix B. It is contravariant with respect to $\sigma$ and hermitian, i.e.

$$
(X Y, Z)_{\omega}=(Y, \sigma(X) Z)_{\omega}, \quad(X, Y)_{\omega}=\eta^{l}(Y, X)_{\omega}^{*}, \quad l \in \mathbb{Z} .
$$

In general, however, the quadratic form (B.11) is not positive semi-definite, and cannot expected to be so on the grounds of (2.28).

We call the $T$-invariant form $\omega_{\beta}$ positive if the associated quadratic form (B.11) is positive definite, i.e. $(X, X)>0$, for all non-zero elements $X$ of the form (B.10). Heuristically one expects $\omega_{\beta}$ to be positive precisely when the local operator whose form factors the sequence $f^{(n)}$ represents is positive. Notice that we require non-degeneracy only for elements of the form (B.10). On elements involving $T^{ \pm}$generators the quadratic form (B.11) is inevitably degenerate due to the $T$-invariance condition (2.21). In addition to these obvious degeneracies there are also some induced by the following relations:

$$
\begin{aligned}
\omega_{\beta}\left(Y C_{a b}(\theta) X\right) & =C_{a b} \omega_{\beta}(Y X), \\
\omega_{\beta}\left(Y C^{a b}(\theta) X\right) & =C^{a b} \omega_{\beta}(Y X), \\
\omega_{\beta}\left(Y T^{-}(\theta \pm i 2 \pi)_{a}^{b} X\right) & =\omega_{\beta}\left(Y T^{-}(\theta)_{a}^{b} X\right), \\
\omega_{\beta}\left(Y T^{+}(\theta \pm i 2 \pi)_{a}^{b} X\right) & =\omega_{\beta}\left(Y T^{+}(\theta)_{a}^{b} X\right),
\end{aligned}
$$

for elements $X, Y \in \mathcal{F}_{\beta}(S)$ with rapidities separated from $\theta$. The first two equations follow from (2.6), (2.5) and (T3), the second pair then follows from (2.7). In particular (2.30) means that within $T$-invariant functionals the operators $C_{a b}(\theta)$ and $C^{a b}(\theta)$ (which are not central) can be replaced with $C_{a b}$ and $C^{a b}$, respectively (which are central). Similarly the action of $T^{ \pm}(\theta)$ within $T$-invariant functionals is $2 \pi i$-periodic even when the $T^{ \pm}(\theta)$ are not periodic.

Let us now address the issue under what conditions a $T$-invariant functional can be written as a vector functional (2.22). Starting with a positive $T$-invariant form one clearly expects this to be the case. This is because one then is in the typical situation where a GNS construction applies. Of course the GNS theorem does not apply literally (generically one is dealing with unbounded operators and topological notions have only introduced indirectly). Nevertheless the basic construction should still apply and yield a state space $\mathcal{H}_{\omega}$ with cyclic vector $\Omega_{\omega}$ and a representation $\pi_{\omega}$ of $\mathcal{F}_{\beta}(S)$ acting on it. The vector $\Omega_{\omega}$ then is the mathematically well-defined version of the symbolic expression $\left(e^{2 \pi K} \mathcal{O}\right)^{1 / 2}$, where $\mathcal{O}$ is a positive local operator in the QFT and $K$ is the generator of Lorentz boosts. Of course positivity of $\omega$ here is essential because only then the state space $\mathcal{H}_{\omega}$ will inherit the positivity of (B.11). In the representation $\pi_{\omega}$ other local operators, not necessarily positive, should have a well-defined action on $\mathcal{H}_{a}$. Of course the inner product of two vectors generated thereby can no longer directly set into correspondence to a form factor. A way to maintain a correspondence to form factors would be to sacrifice positivity of the state space and to take (2.23) as the defining relation for a vector $\left|\Omega_{\beta}\right\rangle$, whether or not the associated functional (2.22) is positive. The state space associated with $\left|\Omega_{\beta}\right\rangle$ then is 


$$
\Sigma=\bigoplus_{n \geqslant 0} \Sigma^{(n)}, \quad \Sigma^{(n)}=\bigoplus_{m \leqslant n} \mathcal{A}^{(m, n)}\left|\Omega_{\beta}\right\rangle
$$

and coincides with $\mathcal{H}_{\omega}$ only when $X \rightarrow\left\langle\Theta_{\beta}|X| \Omega_{\beta}\right\rangle$ is positive.

\section{Modular structures}

In this section algebraic counterparts $(j, \delta)$ of the modular structures featuring in the Tomita-Takesaki theory are derived. As explained in the Introduction, they neatly unravel the 'finite temperature structure' underlying the form factor approach. The modular structures $(j, \delta)$ will be affiliated with the quantum operator algebras $\mathcal{M}_{\beta}(S)$ or $\mathcal{F}_{\beta}(S)$ containing a generalized quantum double $\mathcal{T}_{\beta}(S)$ and a ZF-type algebra $\mathcal{W}(S)$ as distinguished subalgebras. Both are linked, in particular, by the crucial "modular" relation $(\mathrm{M})$. Roughly speaking the quantum double $\mathcal{T}_{\beta}(S)$ can be regarded as "unphysical' in that its elements can be eliminated from expectation values with $T$-invariant functionals. The $\mathcal{W}(S)$ subalgebra in contrast is 'physical' in that its expectation values define the form factors (or solutions of the equivariance equations (I) in the case of $\mathcal{M}_{\beta}(S)$ ). From the viewpoint of the cyclic form factor equation, one of the goals of the formalism here is to make sense out of thermal expectation values over $\mathcal{W}(S)$ with the unbounded 'density operator' $\Delta=e^{\beta K}$, where $K$ is the generator of Lorentz boosts. In upshot, the modular relation $(\mathrm{M})$ allows one to do precisely this. Namely to implement Lorentz boosts with imaginary parameter on $\mathcal{W}(S)$ in terms of the 'unphysical' $\mathcal{T}_{\beta}(S)$ generators, such that in particular the thermal state condition comes out correctly.

In more detail consider the following abelian automorphism group on $\mathcal{W}(S)$

$$
\delta_{n} W_{a}(\theta)=W_{a}(\theta-i \beta n), \quad n \in \frac{1}{2} \mathbb{Z}, \quad \delta_{n} \delta_{m}=\delta_{n+m}=\delta_{m} \delta_{n} .
$$

We set $\delta:=\delta_{1}$. Formally $\delta_{n}=D_{-i n \beta}$, if $D_{\lambda}\left(W_{a}\right)(\theta)=e^{i \lambda K} W_{a}(\theta) e^{-i \lambda K}, \lambda \in \mathbb{R}$ are the Lorentz boosts. The main results are

(a) $\delta_{n} W_{a}(\theta), n \in \frac{1}{2} \mathbb{Z}$, can be implemented in terms of the $\mathcal{T}_{\beta}(S)$ generators.

(b) The algebras $\mathcal{M}_{\beta}(S)$ and $\mathcal{F}_{\beta}(S)$ both 'decompose' into a subalgebra $\mathcal{N}$ and its commutant $\mathcal{N}^{\prime}$, which are related by an involution $j$ (Theorems $2 \mathrm{~A}$ and $2 \mathrm{~B}$ ). $\mathcal{N}$ contains $\mathcal{W}(S)$ as a subalgebra.

(c) The operators $(j, \delta)$ have all algebraic features of "modular structures" in the context of the Tomita-Takesaki theory (Theorem 2C). The counterpart of the "KMS property" is

$$
\omega_{\beta}\left(Y \delta_{1} X\right)=\eta^{l} \omega_{\beta}(X Y), \quad X, Y \in \mathcal{N}, \quad l \in \mathbb{Z},
$$

and generalizes the cyclic form factor equation.

Let us add a few remarks. The 'doubling of the degrees of freedom' in (b) is characteristic of finite temperature equilibrium dynamics. To avoid a possible misunderstanding let us emphasize that this phenomenon is completely unrelated to the description of the $W$-generators in terms of $W_{a}^{+}(\theta)$ and $W_{a}^{-}(\theta)$ operators, which is just a convenient way 
to induce the contraction products $(2.16),(2.17)$ in $\mathcal{F}_{\beta}(S)$, and ultimately the residue equations for the form factors. All of the mentioned results hold also for the algebra $\mathcal{M}_{\beta}(S)$ without any contraction product for the $W$-generators specified.

Probably we should also repeat that one is not dealing with von Neumann algebras. The "modular structures" described here can therefore not directly be subsumed into the framework of the Tomita-Takesaki theory (although this may turn out to be the case in an appropriate reformulation). Nevertheless the "modular structures" described here have all the algebraic features typical for modular structures in the context of von Neumann algebras. Keeping in mind this disclaimer our borrowing of the terms "modular conjugation" and "modular operator" should not give rise to confusion.

Finally it may be worthwhile to point out the similarity to the "corner transfer matrix formalism" used in the context of integrable lattice models. A remarkable feature of the modular operator $\Delta$ in the Tomita-Takesaki theory is that it plays a double role. On the one hand it implements the KMS condition via $(\Omega, Y \Delta X \Omega)=(\Omega, X Y \Omega), X, Y \in \mathcal{M}$. On the other hand it can be used to define a unitary automorphism group, the modular 'time evolution', which leaves the algebra $\mathcal{M}$ and its commutant $\mathcal{M}^{\prime}$ separately invariant. Defining $K \sim \ln \Delta$ to be the "modular hamiltonian" one has $D_{i \lambda}(X)=e^{i \lambda K} X e^{-i \lambda K}, \lambda \in$ $\mathbb{R}$, for this automorphism group. In the context of form factors, the von Neumann algebra $\mathcal{M}$ is replaced with the quantum operator algebra $\mathcal{N}$ and the group $X \rightarrow \Delta^{n} X \Delta^{-n}$ ( $X$ analytic with respect to $\mathrm{Ad} \Delta$ ) corresponds to $\delta_{n}$ in (3.1). According to (a) the latter can be implemented in terms of the generalized quantum double $\mathcal{T}_{\beta}(S)$. The full analogue of the Tomita-Takesaki theorem would state that $\delta_{i \lambda / \beta}, \lambda \in \mathbb{R}$, defines an automorphism group for both $\mathcal{N}$ and its commutant $\mathcal{N}^{\prime}$. Let us suppose for the moment that such an analogue has been obtained. Then there are two ways of implementing Lorentz boosts: the 'ordinary one', where $K$ is just a given generator of the ('kinematical') Poincaré group, and a second one via $\delta_{i t}, t \in \mathbb{R}$, that is in terms of the ('dynamical') form factor algebra $\mathcal{F}_{\beta}(S)$. A similar phenomenon has been discovered around 1979 by Baxter [3] in the context of lattice models, where the "corner transfer matrix" [3,8,39] plays the kinematical/dynamical double role of $\delta$. Combined with the powerful techniques now available in the representation theory of infinite-dimensional quantum algebras this lead to considerable progress in this area; see Ref. [19] for an overview. On the QFT level the implementation of Lorentz boosts with imaginary parameter in terms of $\mathcal{T}_{\beta}(S)$ may be viewed as a counterpart of the corner transfer matrix formalism.

\subsection{Subalgebra $\mathcal{N}$}

As mentioned before the algebras $\mathcal{M}_{\beta}(S)$ and $\mathcal{F}_{\beta}(S)$ differ only insofar as in the latter the contraction product of two $W$-generators is declared by (2.16) and (2.17). For the subalgebras $\mathcal{N} \subset \mathcal{M}_{\beta}(S)$ and $\mathcal{N} \subset \mathcal{F}_{\beta}(S)$ described below we therefore use the same symbol, keeping in mind that the latter differs only by one extra relation, here denoted by (NR), from the former. Of course, $\mathcal{N}$ in both cases is a shorthand for $\mathcal{N}_{\beta}(S)$. 
We denote by $\mathcal{N} \subset \mathcal{M}_{\beta}(S)$ the subalgebra generated by $W_{a}(\theta), D_{a b}^{+}(\theta)$ and $C_{a b}(\theta)$, as defined in (2.3) and (2.14). The fact that these elements form a subalgebra is manifest from the following relations:

$$
\begin{aligned}
& S_{a b}^{m n}\left(\theta_{21}\right) S_{m c}^{k l}\left(\theta_{21}+i \beta-i \pi\right) D_{n k}^{+}\left(\theta_{1}\right) D_{l d}^{+}\left(\theta_{2}\right) \\
& \quad=S_{c d}^{m n}\left(\theta_{12}\right) S_{b n}^{k l}\left(\theta_{12}+i \beta-i \pi\right) D_{a l}^{+}\left(\theta_{2}\right) D_{k d}^{+}\left(\theta_{1}\right), \\
& S_{a b}^{m n}\left(\theta_{21}+i \pi\right) S_{c n}^{k l}\left(\theta_{21}\right) D_{d l}^{+}\left(\theta_{1}\right) C_{k m}\left(\theta_{2}\right) \\
& \quad=S_{m a}^{k l}\left(\theta_{12}+i \beta-i 2 \pi\right) S_{d c}^{m n}\left(\theta_{12}+i \beta-i \pi\right) C_{n l}\left(\theta_{2}\right) D_{k b}^{+}\left(\theta_{1}\right), \\
& S_{a b}^{m n}\left(\theta_{21}\right) S_{c n}^{k l}\left(\theta_{21}-i \pi\right) C_{d l}\left(\theta_{1}\right) C_{k m}\left(\theta_{2}\right) \\
& \quad=S_{m c l}^{k l}\left(\theta_{12}-i \pi\right) S_{d c}^{m n}\left(\theta_{12}\right) C_{n l}\left(\theta_{2}\right) C_{k b}\left(\theta_{1}\right) . \\
& C^{m m} D_{n k}^{ \pm}(\theta) D_{l m}^{\mp}(\theta+i \pi) C^{k l}=\operatorname{dim} V, \quad C^{m n} C_{m n}(\theta)=\operatorname{dim} V,
\end{aligned}
$$

where $D_{a b}^{-}(\theta)$ is the shorthand (2.14). Further, (WW) and

$$
\begin{aligned}
& S_{a b}^{m n}\left(\theta_{21}+i \beta-i 2 \pi\right) D_{c n}^{+}\left(\theta_{1}\right) W_{m}\left(\theta_{2}\right)=S_{c a}^{m n}\left(\theta_{12}+i \pi\right) W_{n}\left(\theta_{2}\right) D_{m b}^{+}\left(\theta_{1}\right), \\
& S_{a b}^{m m}\left(\theta_{21}+i \beta-i 3 \pi\right) C_{c n}\left(\theta_{1}\right) W_{m}\left(\theta_{2}\right)=S_{a c}^{m n}\left(\theta_{12}-i \beta+i 2 \pi\right) W_{m}\left(\theta_{2}\right) C_{m b}\left(\theta_{1}\right),
\end{aligned}
$$

(NW)

where (N1) and (NW) both hold for all relative rapidities. The analogue of (M) is

$$
\begin{aligned}
W_{a}(\theta) & =-\frac{1}{\operatorname{dim} V} C^{m n} D_{a m}^{-}(\theta+i \beta-i 2 \pi) W_{n}(\theta+i \beta), \\
W_{a}(\theta+i \beta) & =-\frac{1}{\operatorname{dim} V} C^{m n} W_{m}(\theta) D_{n a}^{-}(\theta+i \beta-i \pi) .
\end{aligned}
$$

For the action of $\sigma$ one finds

$$
\sigma D_{a b}^{+}(\theta)=D_{b a}^{+}\left(\theta^{*}+i \beta-i 2 \pi\right), \quad \sigma C_{a b}(\theta)=C_{b a}\left(\theta^{*}+2 i \beta-4 i \pi\right),
$$

so that $\mathcal{N}$ is also a $*$-subalgebra of $\mathcal{M}_{\beta}(S)$.

The subalgebra $\mathcal{N} \subset \mathcal{F}_{\beta}(S)$ is again the subalgebra generated by $W_{a l}(\theta), D_{a b}^{+}(\theta)$ and $C_{a b}(\theta)$. They clearly again satisfy the relations (NI), (N2), (NW), (WW) and (NM), but in addition also the relations $(2.16),(2.17)$ for the contraction product of two $W$-generators, which we shall refer to as (NR) in this context.

Before proceeding let us remark that the form factor equations (I), (II) could not have been formulated for functionals over the subalgebra $\mathcal{N}$ only. The appropriate invariance condition on such functionals still would have to guarantee that they are fully determined by their values on strings of $W$-generators. The $T$-invariance condition (2.21) used in Section 2 is too weak. (For example a $T^{-}$generator arising through $\omega_{\beta}\left(X D_{a b}^{+}(\theta)\right)=$ $C_{b b^{\prime}} \omega_{\beta}\left(X T^{-}(\theta+i \pi)_{a}^{b^{\prime}}\right)$ is not an element of $\mathcal{N}$ and cannot be pushed to the left by using the relations of $\mathcal{N}$ only.) On the other hand, a stronger invariance condition adapted to (NM) would no longer reproduce the deformed KZE. We will see below that both $\mathcal{N}$ and its commutant in $\mathcal{F}_{\beta}(S)$ or $\mathcal{M}_{\beta}(S)$ are needed. 


\subsection{Modular conjugation and modular operator}

Let $\overline{\mathcal{F}}_{\beta}^{ \pm}(S)$ denote the auxiliary algebra $\mathcal{F}_{\beta}^{ \pm}(S)$ where the 'structure constants' are replaced with their complex conjugates, i.e.

$$
S_{a b}^{d c}(\theta) \longrightarrow\left[S_{a b}^{d c}(\theta)\right]^{*}, \quad \lambda^{ \pm} \longrightarrow\left(\lambda^{ \pm}\right)^{*}=-\lambda^{ \pm},
$$

keeping in mind the choice $\lambda^{-}=-2 i, \lambda^{+}=-2 i / \operatorname{dim} V$. Further let $\overrightarrow{\mathcal{N}} \subset \overline{\mathcal{F}}_{\beta}(S) \subset$ $\overline{\mathcal{F}}_{\beta}^{ \pm}(S)$ denote the counterpart of the subalgebra $\mathcal{N} \subset \mathcal{F}_{\beta}(S)$. Similarly let $\overline{\mathcal{M}}_{\beta}(S)=$ $\mathcal{M}_{\beta}\left(S^{*}\right)$ be the modular algebra with the complex conjugate $S$-matrix and $\overrightarrow{\mathcal{N}} \subset \overline{\mathcal{M}}_{\beta}(S)$ the corresponding subalgebra. Of course one expects that the original algebras and their 'barred' counterparts are basically the same, i.e. isomorphic. Indeed, a trivial isomorphism is the one that acts as the identity on operators and as complex conjugation on complex numbers. A much more interesting isomorphism is described in Theorem $2 \mathrm{~A}$; what makes it interesting is the content of parts $\mathrm{B}$ and $\mathrm{C}$ of Theorem 2.

To describe this isomorphism let us prepare extra symbols $\bar{W}_{a}^{ \pm}(\theta), \bar{T}^{ \pm}(\theta)_{a}^{b}$ for the generators of $\overline{\mathcal{F}}_{\beta}^{ \pm}(S)$. Similarly we use $\bar{W}_{a}(\theta)=\bar{W}_{a}^{+}(\theta)+\bar{W}_{a}^{-}(\theta)$ for the $W$ generators of $\overline{\mathcal{F}}_{\beta}(S)$ or $\overline{\mathcal{M}}_{\beta}(S)$ and $\bar{D}_{a b}^{+}(\theta)$ and $\bar{C}_{a b}(\theta)$ for the additional generators of the respective $\overline{\mathcal{N}}$ subalgebras. Define an anti-linear operator $j$ acting on $\mathcal{F}_{\beta}^{ \pm}(S)$ by

$$
\begin{aligned}
j T^{+}(\theta)_{a}^{b} & =C_{a a^{\prime}} C^{b b^{\prime}} T^{+}\left(\theta^{*}+\frac{3}{2} i \beta-i 2 \pi\right)_{b^{\prime}}^{a^{\prime}}, \\
j T^{-}(\theta)_{a}^{b} & =C_{a a^{\prime}} C^{b b^{\prime}} T^{-}\left(\theta^{*}+\frac{1}{2} i \beta\right)_{b^{\prime}}^{a^{\prime}}, \\
j W_{a}^{ \pm}(\theta) & =C_{a a^{\prime}} C^{m n} W_{m}^{ \pm}\left(\theta^{*}-\frac{1}{2} i \beta+i \pi\right) T^{+}\left(\theta^{*}+\frac{1}{2} i \beta\right)_{n}^{a^{\prime}} \\
& =C_{a a^{\prime}} C^{m n} T^{-}\left(\theta^{*}+\frac{1}{2} i \beta\right)_{n}^{a^{\prime}} W_{m}^{ \pm}\left(\theta^{*}+\frac{1}{2} i \beta+i \pi\right),
\end{aligned}
$$

and by

$$
j(X Y)=j(X) j(Y), \quad j(z X)=z^{*} j(X), \quad z \in \mathbb{C}
$$

on products of generators. Clearly, (3.5), (3.6) and $j\left(W_{a}(\theta)\right):=j\left(W_{a}^{+}(\theta)+W_{a}^{-}(\theta)\right)$ also defines the action of $j$ on $\mathcal{F}_{\beta}(S)$ and $\mathcal{M}_{\beta}(S)$.

Theorem 2A:

(1) $j: \mathcal{F}_{\beta}^{ \pm}(S) \longrightarrow \overline{\mathcal{F}}_{\beta}^{ \pm}(S)$ defines an anti-linear isomorphism via

$$
\bar{W}_{a}^{ \pm}(\theta)=j W_{a}^{\mp}(\theta), \quad \bar{T}^{ \pm}(\theta)_{a}^{b}=j T^{ \pm}(\theta)_{a}^{b},
$$

and is an involution, i.e. $j^{2}=\mathrm{id}$.

(2) $j: \mathcal{M}_{\beta}(S) \longrightarrow \overline{\mathcal{M}}_{\beta}(S)$ and $j: \mathcal{F}_{\beta}(S) \longrightarrow \overline{\mathcal{F}}_{\beta}(S)$ are anti-linear isomorphisms of $*$-algebras and involutions.

(3) $j: \mathcal{N} \longrightarrow \overline{\mathcal{N}}$ is an anti-linear isomorphism of *-subalgebras for both $\mathcal{N} \subset$ $\mathcal{M}_{\beta}(S)$ and $\mathcal{N} \subset \mathcal{F}_{\beta}(S)$.

Proof. (1) One has to check that $\bar{W}_{a l}^{ \pm}(\theta)$ and $\bar{T}^{ \pm}(\theta)_{a}^{b}$ as defined through (3.5b), (3.7) satisfy all the relations of $\overline{\mathcal{F}}_{\beta}^{ \pm}(S)$. In particular, $j W_{a}(\theta)$ and $j T^{ \pm}(\theta)_{a}^{b}$ with $j W_{a}(\theta)$ 
standing for either $j W_{a}^{+}(\theta)$ or $j W_{a}^{-}(\theta)$ or their sum have to satisfy the relations of $\overline{\mathcal{M}}_{\beta}(S)$. This can be verified by direct although tedious computation. It remains to check the contraction products for the $W$-generators. Using the definitions (3.5b) of $j W_{a}^{ \pm}(\theta)$ and $j T^{ \pm}(\theta)_{a}^{b}$ one computes

$$
\begin{aligned}
j W_{a}^{-}(\theta-i \pi) \cdot j W_{b}^{-}(\theta) & =\lambda^{+} j C_{a b}(\theta+i \beta-i 3 \pi), \\
j W_{a}^{+}(\theta+i \pi) \cdot j W_{b}^{+}(\theta) & =\lambda^{-} C_{a b} .
\end{aligned}
$$

With the identifications $(3.5 b),(3.7)$ and

$$
\begin{aligned}
\bar{C}_{a b}(\theta) & =C_{m n} \bar{T}^{+}(\theta)_{a}^{m} \cdot \bar{T}^{+}(\theta+i \pi)_{b}^{n} \\
& =j C_{a b}(\theta)=C_{a a^{\prime}} C_{b b^{\prime}} C^{a^{\prime} b^{\prime}}\left(\theta^{*}+\frac{3}{2} i \beta-i 2 \pi\right)
\end{aligned}
$$

these are the contraction products for $\overline{\mathcal{F}}_{\beta}^{ \pm}(S)$, as asserted. Consistency requires that also the 'wrong-order' residue conditions come out correctly. Indeed, using again the definitions $(3.5 \mathrm{~b})$ of $j W_{a}^{ \pm}(\theta)$ one can verify

$$
\begin{aligned}
& \bar{W}_{a}^{+}(\theta+i \beta-i \pi) \cdot \bar{W}_{b}^{+}(\theta)=-\lambda^{+} \operatorname{dim} V j D_{a b}^{+}(\theta+i \beta-2 i \pi), \\
& \bar{W}_{a}^{-}(\theta+i \pi-i \beta) \cdot \bar{W}_{b}^{-}(\theta)=-\frac{1}{\operatorname{dim} V} \lambda^{-} j D_{a b}^{-}(\theta-i \pi) .
\end{aligned}
$$

On the other hand, the same contractions can be computed directly in terms of the barred generators starting from (3.8). The result is (3.10) just with the sign flipped. By (3.6) this is consistent with $(2.13)$ and the identification $\bar{T}^{ \pm}(\theta)_{a}^{b}=j T^{ \pm}(\theta)_{a}^{b}$. Finally the property $j^{2}=\mathrm{id}$ can be verified. The statements (2) and (3) are a direct consequence of (1) and Eqs. (3.8), (3.10).

The most remarkable property of $j$ is that the original generators commute with all their $j$-transformed counterparts. We formulate this as a lemma because we shall later encounter a stronger version thereof.

Lemma 1. The generators of $\mathcal{N}$ and $j(\mathcal{N})=\overline{\mathcal{N}}$ mutually commute, for both $\mathcal{N} \subset$ $\mathcal{M}_{\beta}(S)$ and $\mathcal{N} \subset \mathcal{F}_{\beta}(S)$. Explicitly, for $\operatorname{Re} \theta_{12} \neq 0$

$$
\begin{aligned}
j W_{a}\left(\theta_{1}\right) W_{b}\left(\theta_{2}\right) & =W_{b}\left(\theta_{2}\right) j W_{a}\left(\theta_{1}\right), \\
j W_{a}\left(\theta_{1}\right) D_{b c}^{ \pm}\left(\theta_{2}\right) & =D_{b c}^{ \pm}\left(\theta_{2}\right) j W_{a}\left(\theta_{1}\right), \\
j W_{a}\left(\theta_{1}\right) C_{b c}\left(\theta_{2}\right) & =C_{b c}\left(\theta_{2}\right) j W_{a}\left(\theta_{1}\right), \\
j D_{a b}^{ \pm}\left(\theta_{1}\right) D_{c d}^{ \pm}\left(\theta_{2}\right) & =D_{c d}^{ \pm}\left(\theta_{2}\right) j D_{a b}^{ \pm}\left(\theta_{1}\right), \\
j D_{a b}^{ \pm}\left(\theta_{1}\right) C_{c d}\left(\theta_{2}\right) & =C_{c d}\left(\theta_{2}\right) j D_{a b}^{ \pm}\left(\theta_{1}\right), \\
j C_{a b}\left(\theta_{1}\right) C_{c d}\left(\theta_{2}\right) & =C_{c d}\left(\theta_{2}\right) j C_{a b}\left(\theta_{1}\right),
\end{aligned}
$$

where in (b) all combinations of the \pm options are allowed.

The proof is by direct computation. To proceed let $\delta_{n}, n \in \mathbb{Z}$, denote the following discrete automorphism group of $\mathcal{M}_{\beta}(S)$ or $\mathcal{F}_{\beta}(S)$ : 


$$
\begin{aligned}
\delta_{n} T^{ \pm}(\theta)_{a}^{b} & =T^{ \pm}(\theta-i \beta n)_{a}^{b}, \quad \delta_{n} W_{a}(\theta)=W_{a}(\theta-i \beta n), \quad n \in \frac{1}{2} \mathbb{Z}, \\
\delta_{n} \delta_{m} & =\delta_{n+m}=\delta_{m} \delta_{n} .
\end{aligned}
$$

Formally $\delta_{n}=D_{-i n \beta}$ if $D_{\lambda}(X(\theta))=e^{i \lambda K} X(\theta) e^{-i \lambda K}=X(\theta+\lambda), \lambda \in \mathbb{R}$ are the Lorentz boosts. As emphasized in the Introduction, $D_{-i n \beta}$ is however meaningless when defined in terms of the generator $K$ of the Lorentz boosts. On the 'unphysical' $\mathcal{T}_{\beta}(S)$ subalgebra we are free to stipulate that $T^{ \pm}(\theta)_{a}^{b}$ is well defined for generic complex $\theta$. Since they can be eliminated from the 'physical' expectation values $\omega_{\beta}(X)$ no meaning has to be given to $T^{ \pm}(\theta-\operatorname{in} \beta)_{a}^{b}$ in terms of Lorentz boosts with an imaginary parameter. Of course the opposite is true for the subalgebra $\mathcal{W}(S)$ generated by the $W$-operators. In the present framework the action of $D_{-i n \beta}$ on $\mathcal{W}(S)$ can however be implemented in terms of the $\mathcal{T}_{\beta}(S)$ generators, exploiting once more the crucial relation (M). This holds for both the modular algebra $\mathcal{M}_{\beta}(S)$ and the form factor algebra $\mathcal{F}_{\beta}(S)$. Explicitly

$$
\begin{aligned}
\delta_{-1} W_{a}(\theta) & =C_{m n} T^{-}(\theta+i \beta)_{a}^{m} \cdot W_{k}(\theta) \cdot \sigma T^{-}\left(\theta^{*}\right)_{l}^{n} C^{l k}, \\
\delta_{1} W_{a}(\theta) & =C^{k l} \sigma T^{+}\left(\theta^{*}+i \beta\right)_{l}^{n} \cdot W_{k}(\theta) \cdot T^{+}(\theta-i 2 \pi)_{a}^{m} C_{m n},
\end{aligned}
$$

from (2.4) and (2.20). By iteration $\delta_{n}, n \in \mathbb{Z}$, yields a discrete automorphism group of the $\mathcal{W}(S)$ subalgebras of both $\mathcal{F}_{\beta}(S)$ and $\mathcal{M}_{\beta}(S)$.

So far $\delta_{n}$ has only been defined for integer $n$. Also the square root of $\delta_{1}$, say, can be implemented in terms of the $T^{ \pm}$-generators and $j$. It is given by the following alternative expressions:

$$
\delta_{1 / 2} W_{a}(\theta)=\left\{\begin{array}{l}
j W_{n}\left(\theta^{*}+i \pi\right) \cdot T^{+}\left(\theta+\frac{1}{2} i \beta-i 2 \pi\right)_{a}^{n} \\
T^{-}\left(\theta-\frac{1}{2} i \beta\right)_{a}^{n} \cdot j W_{n}\left(\theta^{*}+i \beta+i \pi\right)
\end{array}\right.
$$

Using the relation (M) both expressions can be seen to give $\delta_{1 / 2} W_{a}(\theta)=W_{a}\left(\theta-\frac{1}{2} i \beta\right)$ as required by consistency. In this sense (3.14) it is not an independent automorphism. However, given the $W$-generators in a suitable strip of the complex $\theta$ plane (e.g. $\operatorname{Im} \theta \in$ $[0, \pi]$ for the first expression) as well as $j, \mathrm{Eq} .(3.14)$ can be used to extend the domain of definition by half-integer multiples of $i \beta$, as it should.

The relation of (3.14) to the square root of $\delta$ can also be seen as follows. There exists a linear (not antilinear) anti-automorphism $\bar{s}$ of $\mathcal{F}_{\beta}(S)$ and $\mathcal{M}_{\beta}(S)$ given by [28]

$$
\begin{aligned}
\bar{s} T^{+}(\theta)_{a}^{b} & =C_{a a^{\prime}} C^{b b^{\prime}} T^{-}(\theta+i \pi)_{b^{\prime}}^{a^{\prime}}, \\
\bar{s} T^{-}(\theta)_{a}^{b} & =C_{a a^{\prime}} C^{b b^{\prime}} T^{+}(\theta+i \beta-i \pi)_{b^{\prime}}^{a^{\prime}}, \\
\bar{s} W_{a}(\theta) & =W_{m}(\theta) \cdot \bar{s} T^{-}(\theta)_{a}^{m}=\bar{s} T^{+}(\theta+i \beta-2 \pi i)_{a}^{m} \cdot W_{m}(\theta+i \beta) .
\end{aligned}
$$

The proof is by direct computation, i.e. stipulating that $\bar{s}(X Y)=\bar{s}(Y) \bar{s}(X)$ holds, one checks that the transformed generators again satisfy all relations of $\mathcal{F}_{\beta}(S)$ or $\mathcal{M}_{\beta}(S)$. The square of $\bar{s}$ is

$$
\bar{s}^{2} T^{ \pm}(\theta)_{a}^{b}=T^{ \pm}(\theta+i \beta)_{a}^{b}, \quad \bar{s}^{2} W_{a}(\theta)=W_{a}(\theta+i \beta),
$$


suggesting already a relation to the square root of the $\theta \rightarrow \theta \pm i \beta$ automorphism. To unravel it observe that also $\bar{s}$ has the commutant property described in Lemma 1 [28]: the generators of $\mathcal{N}$ and $\bar{s}(\mathcal{N})$ mutually commute, for both $\mathcal{N} \subset \mathcal{M}_{\beta}(S)$ and $\mathcal{N} \subset \mathcal{F}_{\beta}(S)$. This ensures consistency of the following relation between $\bar{s}, j$ and $\sigma$ : $\bar{s} \circ \sigma=j \circ \delta_{1 / 2}(*)$, where $\delta_{1 / 2}$ is presently simply a shorthand for the shift operation $\theta \rightarrow \theta-i \beta / 2$. The point here is that $\bar{s}, j$ and $\sigma$ are already known to be genuine (anti-)automorphisms of $\mathcal{M}_{\beta}(S)$ and $\mathcal{F}_{\beta}(S)$, so that solving (*) for $\delta_{1 / 2}$ yields the searched for automorphism

$$
\delta_{1 / 2}=j \circ \bar{s} \circ \sigma
$$

Evaluating it on $W_{a}(\theta)$ using $(2.20),(3.15)$ and (3.5b) gives (3.14). For the $\mathcal{T}_{\beta}(S)$ generators one obtains $\delta_{1 / 2} T^{ \pm}(\theta)_{a}^{b}=T^{ \pm}(\theta-i \beta / 2)_{a}^{b}$ as it should. Consistency requires that $\left(\delta_{1 / 2}\right)^{2}=\delta_{1}$, which follows from $\sigma \circ \bar{s} \circ \sigma=\bar{s} \circ \delta_{1}=\bar{s}^{-1}=j \circ \bar{s} \circ j$.

In summary, a discrete abelian automorphism group $\delta_{n}, n \in \frac{1}{2} \mathbb{Z}$, on $\mathcal{M}_{\beta}(S)$ and $\mathcal{F}_{\beta}(S)$ has been defined that implements 'Lorentz boosts with imaginary parameter' $D_{-i n \beta}$ on the 'physical' $\mathcal{W}(S)$ subalgebra consistently in terms of the 'unphysical' $\mathcal{T}_{\beta}(S)$ generators. The $T$-invariance condition (2.21) can now be re-interpreted as the invariance under this discrete automorphism group

$$
\omega_{\beta}\left(\delta_{n}(X)\right)=\eta^{l} \omega_{\beta}(X), \quad n \in \frac{1}{2} \mathbb{Z}
$$

for some $l \in \mathbb{Z}$. For integer $n$ this follows algebraically from the defining relations, for half-integer $n$ it is a consequence of the postulated analyticity properties of (2.24).

Next we turn to the interplay between the automorphism group $\delta_{n}$ and the involution $j$.

Theorem 2B: Let $\omega_{\beta}$ be a $T$-invariant form on either $\mathcal{F}_{\beta}(S)$ or $\mathcal{M}_{\beta}(S)$.

(1) $j \circ \sigma=\sigma \circ j$ and $j \circ \delta_{n}=\delta_{-n} \circ j$ and $\sigma \circ \delta_{n}=\delta_{-n} \circ \sigma$.

(2) $j$ is anti-unitary (up to possibly a phase) with respect to the quadratic form (B.11) induced by $\omega_{\beta}$ and $\sigma$, i.e. $(j(X), j(Y))=\eta^{l}(Y, X), l \in \mathbb{Z}$.

(3) Let $X \in \mathcal{N}$ and $Y$ an element of $\mathcal{F}_{\beta}(S)$ or $\mathcal{M}_{\beta}(S)$ with rapidities separated from that of $X$. Then

$$
\begin{aligned}
\omega_{\beta}\left(Y\left(j \circ \delta_{1 / 2}\right)(X)\right) & =\omega_{\beta}(Y \sigma(X)), \\
\omega_{\beta}\left(\left(j \circ \delta_{-1 / 2}\right)(X) Y\right) & =\eta^{l} \omega_{\beta}(\sigma(X) Y), \quad l \in \mathbb{Z} .
\end{aligned}
$$

Proof. (1) and (3) are verified by direct computation. Let us illustrate (3) for the case of the $\mathcal{W}(S)$ subalgebra of $\mathcal{N}$. To simplify the notation take $\omega_{\beta}$ to be a vector functional built from a $T$-invariant vector $\left|\Omega_{\beta}\right\rangle$. Using the lemma one finds

$$
j\left[W_{a_{1,}}\left(\theta_{n}\right) \ldots W_{a_{1}}\left(\theta_{1}\right)\right]\left|\Omega_{\beta}\right\rangle=W_{a_{1}}\left(\theta_{1}^{*}+i \pi-\frac{1}{2} i \beta\right) \ldots W_{a_{n}}\left(\theta_{n}^{*}+i \pi-\frac{1}{2} i \beta\right)\left|\Omega_{\beta}\right\rangle,
$$

so that $j(X)\left|\Omega_{\beta}\right\rangle=\left(\delta_{1 / 2} \circ \sigma\right)(X)\left|\Omega_{\beta}\right\rangle$ for $X \in \mathcal{W}(S)$. The same can be checked for the generators $C_{a b}(\theta), D_{a b}^{+}(\theta)$ of $\mathcal{N}$ and generic mixed products. Since only 
the 'ket' $T$-invariance condition was used an equivalent way to present the result is $\omega_{\beta}\left(Y\left(j \circ \delta_{1 / 2}\right)(X)\right)=\omega_{\beta}(Y \sigma(X))$, as asserted. For (2) it suffices to consider the case where all rapidities of $X$ are separated from all of $Y$, so that $(Y, X)$ in (B.11) reduces to $\omega_{\beta}(\sigma(Y) X)$. The anti-unitarity of $j$ then amounts to $\omega_{\beta}(j(Z))=\eta^{l} \omega_{\beta}(\sigma(Z))$ with $Z=\sigma(X) Y$, which follows from part (3) and (3.18).

The properties of $j$ and $\delta_{n}$ described in Theorem 2B clearly parallel that of the modular structures $(J, \Delta)$ in the Tomita-Takesaki theory of von Neumann algebras (e.g. as outlined in the Introduction). The identifications are $j(X) \rightarrow J X J$ and $\delta_{n}(X) \rightarrow$ $\Delta^{\prime \prime} X \Delta^{-n}$ ( $X$ analytic with respect to $\operatorname{Ad} \Delta$ ), where $\Delta$ defines the generator of the Lorentz boosts by $\Delta=e^{\beta K}$. Motivated by this analogy we shall refer to $j$ as the modular conjugation and to $\delta$ as the modular operator of the form factor algebra $\mathcal{F}_{\beta}(S)$ or the modular algebra $\mathcal{M}_{\beta}(S)$. The little computation (1.7) establishing the "KMS property" of the modular operator $\Delta$ can directly be taken over: Let $X, Y$ elements of $\mathcal{N}$ with rapidities separated from each other, so that $(Y, X)$ in (B.11) reduces to $\omega_{\beta}(\sigma(Y) X)$. Let $l_{1}, l_{2}$ be suitable integers. Then

$$
\begin{aligned}
\omega_{\beta}\left(Y \delta_{1} X\right) & =\eta^{l_{1}} \omega_{\beta}\left(\delta_{-1 / 2}(Y) \delta_{1 / 2}(X)\right)=\eta^{l_{1}}\left(\sigma \delta_{-1 / 2} Y, j(\sigma X)\right) \\
& =\eta^{l_{1}+l_{2}}\left(\sigma X, j \sigma\left(\delta_{-1 / 2} Y\right)\right)=\eta^{l_{1}+l_{2}} \omega_{\beta}(X Y) .
\end{aligned}
$$

Thus

$$
\omega_{\beta}\left(Y \delta_{1} X\right)=\eta^{l} \omega_{\beta}\left(\left(\delta_{-1} Y\right) X\right)=\eta^{l^{\prime}} \omega_{\beta}(X Y), \quad X, Y \in \mathcal{N}
$$

with $l, l^{\prime} \in \mathbb{Z}$. In particular for $Y=W_{a_{n}}\left(\theta_{n}\right), X=W_{a_{n-1}}\left(\theta_{n-1}\right) \ldots W_{a_{1}}\left(\theta_{1}\right)$ one recovers the cyclic form factor equation. Of course this is also follows directly from (M) and (2.21) (see Theorem 1 and Ref. [28]), but it is gratifying to see it reappear from the underlying 'finite temperature' automorphism structure, without having to appeal to the deformed KZE equation.

In order to complete the analogy with the Tomita-Takesaki theory, the image $j(\mathcal{N})$ of the subalgebra $\mathcal{N}$ should coincide with the commutant of $\mathcal{N}$ in $\mathcal{F}_{\beta}(S)$ or $\mathcal{M}_{\beta}(S)$. A little technical problem here is that $j(\mathcal{N})$ lives in $\overline{\mathcal{F}}_{\beta}(S)$ or $\overline{\mathcal{M}}_{\beta}(S)$, while $\mathcal{N}$ is a subalgebra of the 'unbarred' algebras. This can easily be rectified by concatenating $j$ with the trivial anti-linear automorphism $\iota$ acting like the identity on algebra elements and like complex conjugation on complex numbers. Denoting by $J=\iota 0 j$ this concatenation, $J(\mathcal{N})$ is a subalgebra of $\mathcal{F}_{\beta}(S)$ or $\mathcal{M}_{\beta}(S)$ of which one can ask how it relates to the original subalgebra $\mathcal{N}$. Next one has to specify what one means by the commutant of $\mathcal{N}$ in this context. Naturally one will require that $\mathcal{N}^{\prime}$ is again a quantum operator algebra generated from degree- 1 elements $X(\theta)$ by the two multiplication operations. Further it should be the largest quantum operator subalgebra of $\mathcal{F}_{\beta}(S)$ or $\mathcal{M}_{\beta}(S)$ commuting with $\mathcal{N}$. With these specifications the following result holds.

Theorem $2 C$ : Let $\mathcal{N}^{\prime}$ denote the commutant of $\mathcal{N}$ in $\mathcal{F}_{\beta}(S)$. Then $\mathcal{N}^{\prime}$ is a $*$-subalgebra of $\mathcal{F}_{\beta}(S)$ isomorphic to $J(\mathcal{N})$. The same holds for $\mathcal{N} \subset \mathcal{M}_{\beta}(S)$. 
Proof. Lemma 3 implies that $J W_{a}(\theta), J D_{a b}^{ \pm}(\theta)$ and $\jmath C_{a b}(\theta)$ and hence all finite products thereof are elements of the commutant of $\mathcal{N}$, symbolically $\jmath(\mathcal{N}) \subset \mathcal{N}^{\prime}$. Theorem $2 \mathrm{~A}$ implies that they generate a quantum operator algebra isomorphic to $\iota(\overline{\mathcal{N}})$. On the other hand, the definitions $(2.23),(3.5 \mathrm{~b})$ entail that $\mathcal{N}$ and $J(\mathcal{N})$ generate the same state space (2.31). Since $J(\mathcal{N}) \subset \mathcal{N}^{\prime}$ the same holds a fortiori for $\mathcal{N}^{\prime}$. Thus

$$
\Sigma=\mathcal{N}\left|\Omega_{\beta}\right\rangle=\left({ }_{j} \mathcal{N}\right)\left|\Omega_{\beta}\right\rangle=\mathcal{N}^{\prime}\left|\Omega_{\beta}\right\rangle
$$

From here on one can adopt a standard argument to conclude that

$$
l(\mathcal{N})=\mathcal{N}^{\prime} \quad \text { (as sets) }
$$

i.e. all elements of $\mathcal{N}^{\prime}$ arise as images under $j$ : Set $s=j \circ \delta_{1 / 2}$, which is an algebrahomomorphism and can be checked to satisfy $s^{2}=$ id and $s(X)\left|\Omega_{\beta}\right\rangle=\sigma(X)\left|\Omega_{\beta}\right\rangle$, for all $X \in \mathcal{N}$. By (3.22) one knows that for any $X \in \mathcal{N}$ there exists an $X^{\prime} \in \mathcal{N}^{\prime}$ such that $\sigma(X)\left|\Omega_{\beta}\right\rangle=X^{\prime}\left|\Omega_{\beta}\right\rangle$. For any $Y \in \mathcal{N}$ one then has

$$
s(X) Y\left|\Omega_{\beta}\right\rangle=s(X \sigma(Y))\left|\Omega_{\beta}\right\rangle=Y \sigma(X)\left|\Omega_{\beta}\right\rangle=Y X^{\prime}\left|\Omega_{\beta}\right\rangle=X^{\prime} Y\left|\Omega_{\beta}\right\rangle .
$$

Hence $s \mathcal{N} \subset \mathcal{N}^{\prime}$ and by a symmetric argument $s \mathcal{N}^{\prime} \subset \mathcal{N}$. It follows that $s \mathcal{N}=\mathcal{N}^{\prime}$ and thus also that $J(\mathcal{N})$ and $\mathcal{N}^{\prime}$ coincide as sets. Since $J(\mathcal{N})=\iota(\overline{\mathcal{N}})$ is already known to be a $*$-subalgebra of $\mathcal{F}_{\beta}(S)$ the same holds for $\mathcal{N}^{\prime}$.

Let us emphasize that the above result does not apply to elements of 'infinite degree', which are beyond the scope of the "quantum operator algebra" concept. Theorem $2 \mathrm{C}$ can be regarded as part of the form factor analogue of the Tomita-Takesaki theorem. The second part would consist in showing that starting from the definitions (3.13), (3.14) also $\delta_{i \lambda / \beta}, \lambda \in \mathbb{R}$ can be defined and provides an automorphism group of both $\mathcal{N}$ and $\mathcal{N}^{\prime}$ that coincides with the original Lorentz boosts $D_{\lambda}$. Since $\mathcal{T}_{\beta}(S)$ via the $S$-matrix carries dynamical information, this would reveal part of its 'kinematical-dynamical' double role alluded to in the Introduction to this section.

\subsection{Further properties}

The usefulness of the automorphism

$$
s=j \circ \delta_{1 / 2}=\bar{s} \circ \sigma
$$

encountered twice in the previous section is not accidental. ${ }^{3}$ It is the counterpart of the Tomita operator $\breve{S}$ forming the starting point of the Tomita-Takesaki theory. To explain this, let us briefly recap how the modular operators $(J, \Delta)$ are usually constructed in the context of von Neumann algebras: Let $\mathcal{N}$ be a von Neumann algebra in "standard

\footnotetext{
${ }^{3}$ We apologize for not avoiding the clash of several standard notations here: $s$ must not be confused with the "antipode" in the context of Hopf algebras. The Tomita operator $\check{S}$ of course has nothing to do with the $S$-matrix. Both $s$ and $\check{S}$ in the sense of (3.25), (3.26) appear only within the next two paragraphs. In Section 3.3 we write $\mathcal{N}_{\beta}(S)$ for the quantum operator algebras of Sections 3.1 and 3.2.
} 
form". This means that $\mathcal{N}$ acts on a Hilbert space $\mathcal{H}$ possessing a cyclic and separating vector $\Omega$ and that both $D:=\mathcal{N} \Omega$ and $D^{\prime}:=\mathcal{N}^{\prime} \Omega$ are dense in $\mathcal{H}$. Here as usual $\mathcal{N}^{\prime}$ denotes the commutant of $\mathcal{N}$ in $\mathcal{B}(\mathcal{H})$, the bounded operators on $\mathcal{H}$. Define operators $\check{S}$ and $\check{F}$ by

$$
\begin{array}{ll}
\check{S}: & D \rightarrow D, \quad \check{S} X \Omega=X^{*} \Omega, \quad X \in \mathcal{N}, \\
\check{F}: & D^{\prime} \rightarrow D^{\prime}, \quad \check{F} X^{\prime} \Omega=\left(X^{\prime}\right)^{*} \Omega, \quad X^{\prime} \in \mathcal{N}^{\prime} .
\end{array}
$$

They are closable operators and their closures, also denoted by $\breve{S}$ and $\breve{F}$, admit a polar decomposition

$$
\check{S}=J \Delta^{1 / 2} \quad \text { with } \check{S} \check{F}=\Delta^{-1}, \quad \check{F} \check{S}=\Delta,
$$

where $J$ is anti-unitary with respect to the inner product on $\mathcal{H}$ and $\Delta$ is a positive self-adjoint (in general unbounded) operator. The operators $(J, \Delta)$ are the modular structures featuring in the Tomita-Takesaki theorem. Thus, at least in principle, they can be constructed from the operators $\check{S}, \check{F}$ defined in (3.26).

In the present context $s$ in (3.25) plays the role of $\operatorname{Ad} \check{S}$ in (3.26) and the quantum operator algebra $\mathcal{N}_{\beta}(S)$ of Section 3.2 plays the role of the von Neumann algebra $\mathcal{N}$ in (3.26). Indeed, using the automorphism $\bar{s}$ in (3.15) to define $s=\bar{s} \circ \sigma$ and $f=\sigma \circ \bar{s}$, one verifies the following properties of $s$ and $f$ : Both are anti-linear automorphisms (not anti-automorphisms) of $\mathcal{M}_{\beta}(S)$ or $\mathcal{F}_{\beta}(S)$. They are involutions $s^{2}=i d, f^{2}=$ id and related by $f=\sigma s \sigma$. Further

$$
\begin{aligned}
s \circ f & =\delta_{-1}, \quad f \circ s=\delta_{1}, \\
s(X)\left|\Omega_{\beta}\right\rangle & =\sigma(X)\left|\Omega_{\beta}\right\rangle, \quad X \in \mathcal{N}_{\beta}(S), \\
(s(X), s(Y)) & =\eta^{l}(\sigma(X), \sigma(Y)), \quad l \in \mathbb{Z},
\end{aligned}
$$

where $\left|\Omega_{\beta}\right\rangle$ is a $T$-invariant vector. These properties are completely analogous to that of $X \rightarrow \breve{S} X \check{S}, X \rightarrow \breve{F} X \breve{F}$ with $\breve{S}, \breve{F}$ defined through (3.26). Of course the construction principle (3.27) for the modular structures does not apply here: One is not dealing with von Neumann algebras, the state space (2.31) in general does not carry a positive semi-definite inner product, and the topological structure is lacking. Fortunately this is also not needed. The modular structures $(j, \delta)$ here are defined directly and explicitly in terms of the $\mathcal{T}_{\beta}(S)$ generators. The automorphisms $s$ and $f$ are derived quantities which can also be written down explicitly. In summary, the modular structures $(j, \delta)$ found here and the 'canonical' ones within the Tomita-Takesaki theory can be contrasted as follows.

- The modular structures $(j, \delta)$ are affiliated with "quantum operator algebras" $\mathcal{M}_{\beta}(S)$ or $\mathcal{F}_{\beta}(S)$ containing a generalized "quantum double" $\mathcal{T}_{\beta}(S)$ as a subalgebra.

- They are constructed explicitly in terms of the $\mathcal{T}_{\beta}(S)$ generators, not by means of polar decomposition of a closable operator. 
- The construction does not rely on the existence of positive functionals over the algebra $\mathcal{M}_{\beta}(S)$ or $\mathcal{F}_{\beta}(S)$. The state space $\Sigma$ in (2.31) may, but need not, have a positive semi-definite inner product.

- Topological notions are lacking in the quantum operator algebras $\mathcal{M}_{\beta}(S)$ or $\mathcal{F}_{\beta}(S)$. In particular elements of infinite degree are not defined.

The first three features should probably not be counted as drawbacks, but the last one certainly calls for improvement. This is because local operators in this framework probably are described by elements of infinite degree, having among others the property to map solutions of (2.23) onto new solutions. In the special case, where one starts from a positive functional $\omega_{\beta}$ it is plausible that upon appropriate refinement, the construction of $(j, \delta)$ described here can be subsumed within the framework of the Tomita-Takesaki theory.

So far the algebras $\mathcal{M}_{\beta}(S)$ and $\mathcal{F}_{\beta}(S)$ ran completely parallel as far as the modular structures $(j, \delta)$ were concerned. Of course they are different concerning their role in the form factor construction: The $T$-invariant functionals over $\mathcal{M}_{\beta}(S)$ yield solutions of the equivariance equations (I), while the functionals over the form factor algebra $\mathcal{F}_{\beta}(S)$ yield solutions of the combined system (I) and (II). One may ask however to what extent they are different with respect to the role of the modular structures $(j, \delta)$. We propose the following answer: For $\mathcal{F}_{\beta}(S)$ it is possible to recover the full algebra from the subalgebra $\mathcal{W}(S) \subset \mathcal{N}_{\beta}(S)$ and its commutant $\mathcal{W}(S)^{\prime} \subset \mathcal{N}_{\beta}(S)^{\prime}$, while for $\mathcal{M}_{\beta}(S)$ the same is not possible. Though we cannot offer yet a fully fledged reconstruction theorem, the following features presumably capture the basic ingredients.

We begin by computing the contraction products between the $W^{ \pm}$and the $j W^{ \pm}$ generators. Using (T2), (T3) the conditions ( $\mathrm{R} \pm$ ) translate into

$$
\begin{aligned}
T^{+}(\theta)_{a}^{b} & =-\frac{1}{\lambda^{+}} C^{b b^{\prime}} W_{a}^{+}(\theta-i \beta+i 2 \pi) \cdot j W_{b^{\prime}}^{+}\left(\theta^{*}+\frac{1}{2} i \beta-i 2 \pi\right) \\
& =-\frac{1}{\lambda^{-}} C^{b b^{\prime}} W_{a}^{-}(\theta-i \beta+i 2 \pi) \cdot j W_{b^{\prime}}^{-}\left(\theta^{*}+\frac{1}{2} i \beta\right), \\
T^{-}(\theta)_{a}^{b} & =-\frac{1}{\lambda^{+}} C^{b b^{\prime}} j W_{b^{\prime}}^{+}\left(\theta^{*}+\frac{1}{2} i \beta+i 2 \pi\right) \cdot W_{a}^{+}(\theta) \\
& =-\frac{1}{\lambda^{-}} C^{b b^{\prime}} j W_{b^{\prime}}^{-}\left(\theta^{*}+i \frac{1}{2} \beta\right) \cdot W_{a}^{-}(\theta) .
\end{aligned}
$$

An alternative evaluation using $(\mathrm{R} \pm$ ) and (TW) yields

$$
\begin{aligned}
& T^{+}(\theta)_{a}^{b}=\frac{1}{\lambda^{-}} C^{b b^{\prime}} j W_{b^{\prime}}^{+}\left(\theta^{*}+\frac{1}{2} i \beta\right) \cdot W_{a}^{+}(\theta-i \beta+i 2 \pi), \\
& T^{-}(\theta)_{a}^{b}=\frac{1}{\lambda^{-}} C^{b b^{\prime}} W_{a}^{+}(\theta) \cdot j W_{b^{\prime}}^{+}\left(\theta^{*}+i \frac{1}{2} \beta\right) .
\end{aligned}
$$

Notice that these relations are manifestly consistent with the (TW) relations just because the $W$-generators commute with the $j W$ 's. When the $S$-matrix is $2 \pi i$-periodic a similar computation yields

$$
T^{+}(\theta)_{a}^{b}=\frac{1}{\lambda^{+}} C^{b b^{\prime}} j W_{b^{\prime}}^{-}\left(\theta^{*}+\frac{1}{2} i \beta\right) \cdot W_{a}^{-}(\theta-i \beta)
$$




$$
T^{-}(\theta)_{a}^{b}=\frac{1}{\lambda^{+}} C^{b b^{\prime}} W_{a}^{-}(\theta+i 2 \pi) \cdot j W_{b^{\prime}}^{-}\left(\theta^{*}+i \frac{1}{2} \beta\right) .
$$

One of the steps in the derivation of (3.31) is only valid for a non-singular $S_{a b}^{d c}(-i \pi)$; alternatively (3.31) is clearly consistent with the (TW) relations only if $T^{ \pm}(\theta)$ are $2 \pi i$-periodic.

For the sum $W_{a}(\theta)=W_{a}^{+}(\theta)+W_{a}^{-}(\theta)$ the eq.s $(3.29 \mathrm{db}, \mathrm{d})$ and (3.30) can be combined to

$$
\begin{aligned}
T^{+}(\theta)_{a}^{b} & =-\frac{1}{\lambda^{-}} C^{b b^{\prime}} W_{a}(\theta-i \beta+i 2 \pi) \cdot j W_{b^{\prime}}\left(\theta^{*}+\frac{1}{2} i \beta\right) \\
& =\frac{1}{\lambda^{-}} C^{b b^{\prime}} j W_{b^{\prime}}\left(\theta^{*}+\frac{1}{2} i \beta\right) \cdot W_{a}(\theta-i \beta+i 2 \pi), \\
T^{-}(\theta)_{a}^{b} & =-\frac{1}{\lambda^{-}} C^{b b^{\prime}} j W_{b^{\prime}}\left(\theta^{*}+i \frac{1}{2} \beta\right) \cdot W_{a}(\theta) \\
& =\frac{1}{\lambda^{-}} C^{b b^{\prime}} W_{a}(\theta) \cdot j W_{b^{\prime}}\left(\theta^{*}+i \frac{1}{2} \beta\right) .
\end{aligned}
$$

The remaining equations $(3.29 \mathrm{da}, \mathrm{c})$ yield

$$
\begin{aligned}
& T^{+}(\theta-i 2 \pi)_{a}^{b}=-\frac{1}{\lambda^{+}} C^{b b^{\prime}} W_{a}(\theta-i \beta) \cdot j W_{b^{\prime}}\left(\theta^{*}+\frac{1}{2} i \beta\right), \\
& T^{-}(\theta+i 2 \pi)_{a}^{b}=-\frac{1}{\lambda^{+}} C^{b b^{\prime}} j W_{b^{\prime}}\left(\theta^{*}+\frac{1}{2} i \beta\right) \cdot W_{a}(\theta+i 2 \pi) .
\end{aligned}
$$

One sees that the $\mathcal{T}_{\beta}(S)$ generators can be recovered from that of $\mathcal{W}(S)$ and $j \mathcal{W}(S)$. More generally, the reconstruction theorem envisaged would take two commuting algebras $\mathcal{N}$ as the starting point and show that from suitable contraction products between them the original algebra $\mathcal{F}_{\beta}(S)$ can be reconstructed. This should facilitate the construction of explicit realizations of $\mathcal{F}_{\beta}(S)$.

\section{Conclusions}

Since we surveyed the results already in the Introduction a few comments on the perspective may be appropriate here.

The study of the representation theory as well as the construction of realizations of the algebra $\mathcal{F}_{\beta}(S)$ is an important desideratum. The following prospects however seem to make it worthwhile to consider. The appearance of a double TTS algebra should allow one to make contact to better understood areas like quantum groups and Bethe ansatz techniques $[14,41,2,32]$. The implementation of Lorentz boosts with imaginary parameter in terms of the TTS generators can be viewed as a QFT counterpart of Baxter's corner transfer matrix formalism for integrable models in statistical mechanics $[3,8,39]$. Making this relation precise might in addition be a route to a Euclidean analogue of modular structures.

On an algebraic level we expect that the modular structures $(j, \delta)$ can be used to give an alternative derivation of Smirnov's "local commutativity theorem" [34]. The 
spin-off of emphasizing the modular structures underlying it should be to see how the result generalizes to non-integrable QFTs.

Finally the algebraic framework described here should prepare the ground for generalizations, as mentioned in the Introduction.

\section{Acknowledgements}

Most of this work was done in Kyoto, enjoying the hospitality of the Yukawa Institut. I wish to thank T. Inami, M. Jimbo, H. Konno, V. Korepin, T. Miwa, M. Pillin and R. Sasaki for interesting discussions.

\section{Appendix A. Form factor equations}

Here we summarize our conventions for the form factor equations. We work with a slightly generalized set of form factor equations, depending on a real parameter $\beta$. For $\beta=2 \pi$ they coincide with the form factor equations of an integrable massive QFT without bound states. For generic $\beta$ one obtains a system of deformed form factor equations, whose solutions turn out to define QFTs over some non-commutative space, while leaving the $S$-matrix unchanged [29]. Conceptually the solutions to both systems of equations are sequences of tensor-valued meromorphic functions. The equations are conveniently grouped into two sets (I) and (II). The set (I) is a system of equivariance equations characterizing the individual members of a sequence, while the set (II) prescribes how the solutions of (I) are arranged into sequences.

The input for the (generalized) form factor equations is a given two-particle bootstrap $S$-matrix. To fix our conventions, we repeat the defining relations. A matrix-valued meromorphic function $S_{a b}^{d c}(\theta), \theta \in \mathbb{C}$, is called a two-particle $S$-matrix if it satisfies the following set of equations. First the Yang Baxter equation

$$
S_{a b}^{n m i}\left(\theta_{12}\right) S_{n c}^{k p}\left(\theta_{13}\right) S_{m p}^{j i}\left(\theta_{23}\right)=S_{b c}^{n m}\left(\theta_{23}\right) S_{a m}^{p i}\left(\theta_{13}\right) S_{p n}^{k j}\left(\theta_{12}\right)
$$

where $\theta_{12}=\theta_{1}-\theta_{2}$, etc. Second unitarity (A.2ca,b) and crossing invariance (A.2cc)

$$
\begin{aligned}
S_{a b}^{m n}(\theta) S_{n m}^{c d}(-\theta) & =\delta_{a}^{d} \delta_{b}^{c}, \\
S_{a n}^{m c}(\theta) S_{b m}^{n d}(2 \pi i-\theta) & =\delta_{a}^{d} \delta_{b}^{c}, \\
S_{a b}^{d c}(\theta) & =C_{a a^{\prime}} C^{d d^{\prime}} S_{b d^{\prime}}^{c a^{\prime}}(i \pi-\theta),
\end{aligned}
$$

where (A.2cc) together with one of the unitarity conditions (A.2ca), (A.2cb) implies the other. Further, real analyticity and Bose symmetry,

$$
\left[S_{a b}^{d c}(\theta)\right]^{*}=S_{a b}^{d c}\left(-\theta^{*}\right), \quad S_{a b}^{d c}(\theta)=S_{b a}^{c d}(\theta) .
$$

Finally the normalization condition

$$
S_{a b}^{d c}(0)=-\delta_{a}^{c} \delta_{b}^{d}
$$


It is convenient to borrow Penrose's abstract index notation from general relativity. That is to say, indices $a, b, \ldots$ are not supposed to take numerical values but merely indicate the tensorial character of the quantity carrying it. Vectors $v^{a}, v^{b}, \ldots$ for example are elements of (classes of) abstract modules $V^{a}, V^{b}, \ldots$ of the same dimensionality $\operatorname{dim} V$. Covectors $v_{a}, v_{b}, \ldots$ are elements of the dual modules $V_{a}, V_{b}, \ldots$ and repeated upper and lower case indices indicate the duality pairing. Indices can be raised and lowered by means of the constant 'charge conjugation matrix' $C_{a b}$ and its inverse $C^{a b}$, satisfying $C_{a d} C^{d b}=\delta_{a}^{b}$. The $S$-matrix is a meromorphic function of $\theta$. Bound state poles, if any, are situated on the imaginary axis in the so-called physical strip $0 \leqslant \operatorname{Im} \theta<\pi$. From crossing invariance and the normalization (A.4) one infers that $S_{a b}^{d c}(i \pi)=-C_{a b} C^{c d}$ is always regular, in contrast to $S_{a b}^{d c}(-i \pi)$ which may be singular. In fact, the relevant $S$-matrices are of one of the following two types:

(a) $S_{a b}^{d c}(\theta)$ is $2 \pi i$-periodic, or else

(b) $S_{a b}^{d c}(-i \pi)$ is singular.

The form factor equations are a system of recursive functional equations for tensorvalued meromorphic functions of many variables. In the algebraic formulation adopted here they arise from the $T$-invariant states $(2.21),(2.23)$ and the relations of the algebra $\mathcal{F}_{\beta}(S)$. This gives a system of functional equations for the matrix elements (2.24), whose consistency is ensured by the consistency of the underlying algebra. Both for $\beta=2 \pi$ and $\beta$ generic two systems of equations arise: First a system of equivariance equations $^{4}$ (I) that prescribes their monodromy under the action of an infinite discrete group $W_{n}$ acting on the arguments

$$
f_{A}(\theta)=L_{w}(\theta)_{A}^{B} f_{B}\left(w^{-1} \theta\right), \quad L_{w_{1} w_{2}}(\theta)_{A}^{B}=L_{w_{1} 1}(\theta){ }_{A}^{C} L_{w_{2}}\left(w_{1}^{-1} \theta\right)_{C}^{B} .
$$

Here $L_{w}(\theta)_{A}^{B}=L_{w}\left(\theta_{n}, \ldots, \theta_{1}\right)_{a_{n} \ldots a_{1}}^{b_{n} \ldots b_{1}}$ is the matrix representing $w \in W_{n}$ on the space of $V^{\otimes n}$-valued functions. The group $W_{n}$ is the semidirect product of the permutation group $S_{n}$ and the translation group $\mathbb{Z}^{n}$ [7] and turns out to be generated by only two elements, $s_{1}$ and $\Omega$. Their action on rapidity vectors and the corresponding representation matrices are given by

$$
\begin{array}{ll}
s_{1}\left(\theta_{n}, \ldots, \theta_{1}\right)=\left(\theta_{n}, \ldots, \theta_{3}, \theta_{1}, \theta_{2}\right), & L_{s_{1}}(\theta)_{A}^{B}=\delta_{a_{n}}^{b_{n}} \ldots \delta_{a_{3}}^{b_{3}} S_{a_{2} a_{1}}^{b_{1} b_{2}}\left(\theta_{21}\right), \\
\Omega\left(\theta_{n}, \ldots, \theta_{1}\right)=\left(\theta_{1}+i \beta, \theta_{n}, \ldots, \theta_{2}\right), & L_{\Omega}(\theta)_{A}^{B}=\eta \delta_{a_{n-1}}^{b_{n}} \delta_{a_{n-2}}^{b_{n-1}} \ldots \delta_{a_{1}}^{b_{2}} \delta_{a_{n}}^{b_{1}} .
\end{array}
$$

$W_{n}$ can also be considered as a Coxeter group, in which case the length of a group element $w \in W_{n}$ coincides with the power of $L_{w}(\theta)$ in the two-particle $S$-matrix. $\Omega^{n}$ is a central element of $W_{n}$, which on the functions (I) is represented as $\eta^{n} \delta_{A}^{B}$. Explicitly the equivariance equations (I) for the elements $s_{1}, \Omega \in W_{n}$ are

\footnotetext{
${ }^{4}$ The term is borrowed from Ref. [13].
} 


$$
\begin{aligned}
& f_{a_{n} \ldots a_{1}}\left(\theta_{n} \ldots \theta_{1}\right)=S_{a_{2} a_{1}}^{d c}\left(\theta_{21}\right) f_{a_{n} \ldots a_{n-2} c d}\left(\theta_{n}, \ldots, \theta_{3}, \theta_{1}, \theta_{2}\right), \\
& f_{a_{n} \ldots a_{1}}\left(\theta_{n}+i \beta, \theta_{n-1}, \ldots, \theta_{1}\right)=\eta f_{a_{n-1} \ldots a_{1} a_{n}}\left(\theta_{n-1}, \ldots, \theta_{1}, \theta_{n}\right) .
\end{aligned}
$$

For later use let us also note the representation matrices for the generators $t_{j}, 1 \leqslant$ $j \leqslant n$, of the translation subgroup. Using $t_{j}=s_{j} \ldots s_{n-1} \Omega s_{1} \ldots s_{j-1}, s_{i+1}:=\Omega^{-1} s_{i} \Omega$, $i=1, \ldots, n-2$, one finds from (I) and (A.6)

$$
\begin{aligned}
& t_{j}\left(\theta_{n}, \ldots, \theta_{1}\right)=\left(\theta_{n}, \ldots, \theta_{j}+i \beta, \ldots, \theta_{1}\right), \\
& L_{t_{j}}(\theta)_{A}^{B}=\eta T_{a_{j}}^{c}\left(\theta_{j} \mid \theta_{n}, \ldots, \theta_{j+1}\right){ }_{a_{n} \ldots a_{j+1}}^{b_{1} \ldots b_{j+1}} T_{c}^{b_{j}}\left(\theta_{j}-i \beta \mid \theta_{j-1}, \ldots, \theta_{1}\right)_{a_{j-1} \ldots a_{1}}^{b_{j-1} \ldots b_{1}} .
\end{aligned}
$$

Here

$$
T_{a_{n}}^{b_{n}}\left(\theta_{n} \mid \theta_{n-1}, \ldots, \theta_{1}\right)_{a_{n-1} \ldots a_{1}}^{b_{n-1} \ldots b_{1}}=S_{c_{n-1} a_{n-1}}^{b_{n} b_{n-1}}\left(\theta_{n-1, n}\right) S_{c_{n-1} a_{n-2}}^{c_{n-2} b_{n-2}}\left(\theta_{n-2, n}\right) \ldots S_{a_{n} a_{1}}^{c_{1} b_{1}}\left(\theta_{1, n}\right)
$$

is the monodromy matrix; its trace over $a_{n}=b_{n}$ yields the well-known family of commuting operators on $V^{\otimes(n-1)}$. The property $L_{t_{1} \ldots t_{n}}(\theta)_{A}^{B}=\eta^{n} \delta_{A}^{B}$ reflects the fact that $\Omega^{n}=t_{1} \ldots t_{n}$ is central. The equivariance equations $(\mathrm{I})$ in particular imply that starting with a function $f_{A}(\theta)$ analytic in the domain $\operatorname{Re} \theta_{n}>\ldots>\operatorname{Re} \theta_{1}, 0 \leqslant \operatorname{Im} \theta_{i}<$ $\beta, 1 \leqslant i \leqslant n$, and is equivariant with respect to $W_{n}$ the domain of analyticity extends to $\operatorname{Re} \theta_{k j} \neq 0, \forall k, j$. The equivariance (I) for the translation subgroup are also known as the deformed Knizhnik-Zamolodchikov equation (KZE) $[15,33]$.

The second set of form factor equations are residue conditions (II) prescribing the residues at the simple poles of the solutions of (I). These residues in turn get expressed in terms of solutions of (I), but with a lower particle number: $n-2$ in the case of kinematical poles, considered here. Effectively Eqs. (II) thus serve to arrange the solutions of (I) for varying $n$ into sequences $\left(f^{(n)}\right)_{n \geqslant 1}$ such that consecutive (or next to consecutive) members of a sequence are related by the residue conditions (II). The explicit forms follow from $(2.16),(2.17)$ and correspondingly the cases $\beta$ generic and $\beta=2 \pi$ have to be distinguished,

$$
\begin{aligned}
& \beta \text { generic: } \quad \operatorname{Res} f_{A}^{(n)}\left(\theta_{n}, \ldots, \theta_{j} \pm i \pi, \theta_{j}, \ldots, \theta_{1}\right) \\
&=-\lambda^{\mp} C_{a_{j+1} a_{j}} f_{a_{n} \ldots a_{j+2} a_{j-1} \ldots a_{1}}^{(n-2)}\left(\theta_{n}, \ldots, \theta_{j+2}, \theta_{j-1}, \ldots, \theta_{1}\right), \\
& \beta=2 \pi: \quad \operatorname{Res} f_{A}^{(n)}\left(\theta_{n}, \ldots, \theta_{j}+i \pi, \theta_{j}, \ldots, \theta_{1}\right) \\
&=\left[\lambda^{+} L_{t_{j+1}}\left(\theta_{n}, \ldots, \theta_{j}+i \pi, \theta_{j}, \ldots, \theta_{1}\right)_{A}^{B}-\lambda^{-} \delta_{A}^{B}\right] \\
& \quad \times C_{b_{j+1} b_{j}} f_{b_{n} \ldots b_{j+2} b_{j-1} \ldots b_{1}}^{(n-2)}\left(\theta_{n}, \ldots, \theta_{j+2}, \theta_{j-1}, \ldots, \theta_{1}\right) .
\end{aligned}
$$

We use the notation $\operatorname{Res} f(\theta)=\operatorname{res}_{\theta_{j}=\theta_{j-1}+i \pi} f(\theta)$. The constants $\lambda^{ \pm}$are related by $\lambda^{-}=\lambda^{+} \operatorname{dim} V$. The choice $\lambda^{-}=-i \beta / \pi$ matches the normalization of the one-particle states ${ }_{b}\left\langle\theta_{2} \mid \theta_{1}\right\rangle_{a}=2 \beta \delta_{b c} \delta\left(\theta_{21}\right)$. Comparing the first and the second of Eqs. (II) one sees that for generic $\beta$ the residue equations split up into two sets of equations, where the right-hand sides are independent of $\theta_{j}$. The poles at $\theta_{j+1, j}= \pm i \pi$ and $\theta_{j+1, j}=\mp i(\pi-\beta)$ are split and merge in the limit $\beta \rightarrow 2 \pi$.

In summary, two consecutive members of a sequence of form factors are related by the following condition: $f_{A}^{(n)}(\theta)$ is regular at relative rapidities $\theta_{j+1, j}= \pm i \beta, \beta \neq 2 \pi$, 
and has simple poles at relative rapidities $\theta_{j+1, j}= \pm i \pi$ with the above residues. The equivariance relations (I) lead to further poles at relative rapidities $\theta_{k, j}= \pm i \pi+i p \beta, p \in$ $\mathbb{Z}$, whose residues can be computed from (II). The dependence on $\beta$ in the deformed form factors will usually be suppressed. When needed to distinguish them from the undeformed form factors we shall write $\left(f^{(\beta, n)}\right)_{n \geqslant 0}$ and $\left(f^{(2 \pi, n)}\right)_{n \geqslant 0}$ for the deformed and undeformed ones, respectively. As anticipated by the notation one has

$$
f_{A}^{(2 \pi, n)}(\theta)=\lim _{\beta \rightarrow 2 \pi} f_{A}^{(\beta, n)}(\theta)
$$

To verify this one has to show that the r.h.s. solves the undeformed form factor equations. For the equivariance equations this is obvious. To see that the residue equations come out correctly, first note that the deformed KZE implies that $f_{A}^{(\beta, n)}(\theta)$ also has simple poles at $\theta_{j+1, j}=-i \pi+i \beta$ with residues

$$
\begin{aligned}
\operatorname{Res} & f_{A}^{(\beta, n)}\left(\theta_{n}, \ldots, \theta_{j}-i \pi+i \beta, \theta_{j}, \ldots, \theta_{1}\right) \\
= & \lambda^{+} L_{t_{j+1}}\left(\theta_{n}, \ldots, \theta_{j}-i \pi+i \beta, \theta_{j}, \ldots, \theta_{1}\right)_{A}^{B} \\
& \times C_{b_{j+1} b_{j}} f_{b_{n} \ldots b_{j+2} b_{j-1} \ldots b_{1}}^{(\beta, n-2)}\left(\theta_{n}, \ldots, \theta_{j+2}, \theta_{j-1}, \ldots, \theta_{1}\right) .
\end{aligned}
$$

For $\beta \rightarrow 2 \pi$ the poles at $\theta_{j+1, j}=i \pi$ and $\theta_{j+1, j}=i(\beta-\pi)$ merge. They produce a simple pole again because by assumption $f_{A}^{(\beta, n)}(\theta)$ does not have a pole at $\theta_{j+1, j}=i \beta$. In particular this implies that the residues of the merged poles add up producing the second of Eqs. (II), i.e. the undeformed residue axiom.

\section{Appendix B. Quadratic form on $\mathcal{F}_{\beta}(S)$}

To define the quadratic form $(,)_{\omega}$ obeying (2.28) some preparations are needed. Let $F^{(n)}$ denote the space of functions in $n$ real variables that are permutation equivariant and square integrable, i.e. $z \in F^{(n)}$ iff $^{5}$

$$
\begin{aligned}
z_{A}(\theta) & =L_{s}^{*}(\theta){ }_{A}^{B} z_{B}\left(s^{-1} \theta\right), \quad \forall s \in S_{n}, \\
\|z\|^{2} & :=\int \frac{d^{n} \theta}{(4 \pi)^{n}} z_{a}(\theta)^{*} C^{A B} z_{B}(\theta)<\infty .
\end{aligned}
$$

Here $S_{n} \ni s \rightarrow L_{s}$ is the representation of the permutation group inherited from (A.6). For definiteness let us display the representation matrices for the generators $s_{i}$

$$
L_{S_{i}}(\theta){ }_{A}^{B}:=\delta_{a_{n}}^{b_{n}} \ldots \delta_{a_{i+2}}^{b_{i+2}} S_{a_{i+1} a_{i}}^{b_{i} b_{i+1}}\left(\theta_{i+1, i}\right) \delta_{a_{i-1}}^{b_{i-1}} \ldots \delta_{a_{1}}^{b_{1}}, \quad 1 \leqslant i \leqslant n-1,
$$

from which all others can be computed. The norm in (B.1) comes from an inner product on $F^{(n)}$, which for later use we describe in terms of a distributional kernel:

\footnotetext{
${ }^{5}$ Equivariance with respect to the complex conjugate representation $s \rightarrow L_{s}^{*}(\theta)_{A}^{B}:=\left[L_{s}(\theta){ }_{A}^{B}\right]^{*}$ is imposed in order to have the kernel (B.3bb) equivariant with respect to $L$.
} 


$$
\begin{aligned}
\langle z \mid \tilde{z}\rangle & =\int \frac{d^{n} \omega}{(4 \pi)^{n}} \frac{d^{n} \theta}{(4 \pi)^{n}} z_{B^{\prime}}(\omega)^{*} C^{B^{\prime} B}{ }_{B^{r}}\left\langle\omega^{T} \mid \theta\right\rangle_{A} C^{A A^{\prime}} \tilde{z}_{A^{\prime}}(\theta), \\
{ }_{B}\langle\omega \mid \theta\rangle_{A} & =\frac{(4 \pi)^{n}}{n !} \sum_{s \in S_{n}} L_{s}(\theta){ }_{A}^{C} C_{C B^{T}} \delta^{(n)}\left(\omega^{T}-s^{-1} \theta\right),
\end{aligned}
$$

where

$$
B^{T}=\left(b_{1}, \ldots, b_{n}\right), \quad \omega^{T}=\left(\omega_{1}, \ldots, \omega_{n}\right) .
$$

Using $\left[L_{s}(s \theta)_{A}^{B}\right]^{*}=C_{A A^{\prime}} C^{B B^{\prime}} L_{s^{-1}}\left(\theta^{*}\right)_{B^{\prime}}^{A^{\prime}}$ the kernel (B.3bb) can be checked to be hermitian. For equally ordered rapidity vectors $\omega_{n}>\ldots>\omega_{1}$ and $\theta_{n}>\ldots>\theta_{1}$ only the $s=1$ term survives, and for oppositely ordered ones only the $s=\iota$ term. Here $\iota=$ $s_{n-1} \ldots s_{2} s_{1} s_{2} \ldots s_{n-1}$ is the element of $S_{n}$ (considered as a Coxeter group) of maximal length, acting like $\iota\left(\theta_{n}, \ldots, \theta_{1}\right)=\left(\theta_{1}, \ldots, \theta_{n}\right)$ on rapidity vectors. Averaging (B.3bb) with permutation equivariant test functions in $F^{(n)}$ one checks $\langle z \mid z\rangle=\|z\|^{2}$ and hence positive definiteness. Sequences of functions $z^{(n)} \in F^{(n)}$ can be used to construct the Fock space representation of the ZF algebra [25,29].

Permutation equivariant functions can be multiplied by means of the following

Lemma Bl: Let $g \in F^{(k)}$ and $h \in F^{(n-k)}$ be $L^{*}$-equivariant under the permutation group, i.e.

$$
\begin{array}{ll}
g_{A}(\theta)=L_{s}^{*}(\theta){ }_{A}^{B} g_{B}\left(s^{-1} \theta\right), & s \in S_{k}, \\
h_{A}(\theta)=L_{s}^{*}(\theta){ }_{A}^{B} h_{B}\left(s^{-1} \theta\right), & s \in S_{n-k} .
\end{array}
$$

Let $\left(I_{+}, I_{-}\right)$be a partition of $I=(n, \ldots, 1)$ into ordered subsets $I_{+}=\left(i_{n}, \ldots, i_{n-k+1}\right)$, $i_{n}>\ldots>i_{n-k+1}$ and $I_{-}=\left(i_{n-k}, \ldots, i_{1}\right), i_{n-k}>\ldots>i_{1}$ and $\operatorname{set} \theta_{+}=\left(\theta_{i_{n}}, \ldots, \theta_{i_{n-k+1}}\right)$, $\theta_{-}=\left(\theta_{i_{n-k}}, \ldots, \theta_{i_{1}}\right)$. To each of these $\left(\begin{array}{l}n \\ k\end{array}\right)$ partitions associate an element $s\left(I_{+}, I_{-}\right) \in S_{n}$ by $s\left(I_{+}, I_{-}\right)\left(\theta_{+}, \theta_{-}\right)=\left(\theta_{n}, \ldots, \theta_{1}\right)$. Then the function $g \circ h \in F^{(n)}$ defined by

$$
(g \circ h)_{A}(\theta):=\sum_{\left(I_{+}, I_{-}\right)} L_{s\left(I_{+}, I_{-}\right)}^{*}(\theta)_{A}^{B} g_{b_{n} \ldots b_{n-k+1}}\left(\theta_{+}\right) h_{b_{n-k} \ldots b_{1}}\left(\theta_{-}\right)
$$

is $L^{*}$-equivariant under $S_{n}$. The multiplication ' $O$ ' is distributive, associative but noncommutative. The so-defined algebra of permutation equivariant functions carries a *operation $\sigma_{\circ}$ given by

$$
\left(\sigma_{\circ} g\right)_{A}(\theta)=g_{A^{T}}^{*}\left(\theta^{T}\right), \quad \sigma_{\circ}(g \circ h)=\sigma_{\circ} h \circ \sigma_{\circ} g .
$$

The same of course holds for $L$-equivariant functions.

We omit the proof. Observe that in the trivial case where $S$ is replaced with plus or minus the permutation matrix, the product (B.5) reduces to the moment multiplication or the wedge product, respectively.

For $n, m \geqslant 0$, let now $G^{(k)} \in F^{(k)^{*}}, k=n+m-2 l, l=0, \ldots, \min (m, n)$, be a set of L-equivariant functions. Use the notation of Lemma B1 with $\omega=\left(\omega_{n}, \ldots, \omega_{1}\right), B=$ $\left(b_{m}, \ldots, b_{1}\right)$ and $\theta=\left(\theta_{n}, \ldots, \theta_{1}\right), A=\left(a_{n}, \ldots, a_{1}\right)$. Define the following distributional kernel: 


$$
\begin{aligned}
\bar{G}_{B A}(\omega \mid \theta)= & \sum_{\forall\left(J_{+}, J_{-}\right), \forall\left(I_{+}, I_{-}\right)} L_{s\left(J_{-}, J_{+}\right)}(\omega)_{B C_{1}}^{C}\left\langle\omega_{+} \mid \theta_{+}\right\rangle_{D_{+}} \\
& \times G_{C_{-} D_{-}}\left(\omega_{-}+i \pi, \theta_{-}\right) L_{s\left(I_{+}, I_{-}\right)}(\theta)_{A}^{D} .
\end{aligned}
$$

Here the sum runs over all partitions of $J=(m, \ldots, 1)$ and $I=(n, \ldots, 1)$ into pairs of ordered subsets, not just those with a fixed number of elements $\left|J_{+}\right|$in $J_{+}$or $\left|I_{+}\right|$in $I_{+}$as in Lemma B1. Again the indices are not permuted, i.e. $D_{+}=\left(d_{n}, \ldots, d_{n-\left|I_{+}\right|+1}\right), D_{-}=$ $\left(d_{n-\left|I_{+}\right|}, \ldots, d_{1}\right)$, etc. Finally ${ }_{B}\langle\omega \mid \theta\rangle_{A}$ is the kernel (B.3bb).

Lemma B2: The distribution (B.7) has the following properties.

(a) It is $L$-equivariant in both sets of variables $\omega$ and $\theta$.

(b) It is hermitian in the sense that if there exist $H^{(k)} \in F^{(k)^{*}}$ such that $G_{C}(\theta)^{*}=H_{C^{r}}\left(\theta^{* T}+i \pi\right)$ then

$$
\left[\bar{G}_{B^{T} A}\left(\omega^{T} \mid \theta\right)\right]^{*}=\bar{H}_{A^{T} B}\left(\theta^{T} \mid \omega\right) .
$$

(c) The number of terms in the sum is (B.7) is

$$
\# \text { terms }=\left(\begin{array}{c}
m+n \\
n
\end{array}\right)=\left(\begin{array}{c}
m+n \\
m
\end{array}\right) \text {. }
$$

Proof. (a) Equivariance in $\theta$ follows from Lemma B1, which implies equivariance in $\omega$ once (b) is known. The counting (c) is due to the constraint $\left|J_{+}\right|=\left|I_{+}\right|$. To verify hermiticity (b) it is convenient to make use of the following facts: For any $s \in S_{n}$ the representation matrices $L_{s}$ satisfy $L_{s}^{*}(\iota \theta)_{A^{T}}^{B^{T}}=L_{\iota s \iota}\left(\theta^{*}\right)_{A}^{B}$. Further for any partition $J=\left(J_{+}, J_{-}\right)$of $(n, \ldots, 1)$ there exists a partition $K=\left(K_{+}, K_{-}\right)$of $(n, \ldots, 1)$ with $\left|K_{+}\right|=\left|J_{+}\right|$and $L_{\iota s\left(J_{-}, J_{+}\right) \iota}(\theta)_{A}^{D_{-} D_{+}}=L_{s\left(K_{+}, K_{-}\right)}(\theta)_{A}^{D_{+} D_{-}}$, where $D_{ \pm}$are as above.

Assignments like

$$
F^{(n)} \ni x \longrightarrow W^{n}[x]=\int \frac{d^{n} \theta}{(4 \pi)^{n}} W_{A}(\theta) x_{A^{\prime}}(\theta) C^{A A^{\prime}}
$$

define distributions over $F^{(n)}$. In terms of them we can eventually define the quadratic form on $\mathcal{F}_{\beta}(S)$ associated with a linear form $\omega_{\beta}$. Set

$$
\begin{aligned}
(Y, X)_{\omega}:= & \sum_{\forall\left(I_{+}, I_{-}\right), \forall\left(J_{+}, J_{-}\right)} \int \frac{d^{m} \xi}{(4 \pi)^{m}} \frac{d^{n} \theta}{(4 \pi)^{n}} y_{B^{\prime}}(\xi)^{*} C^{B^{\prime} B} L_{s\left(J_{-}, J_{+}\right)}(\xi)_{B}^{C} \times \\
& \times_{C_{+}}\left\langle\xi_{+} \mid \theta_{+}\right\rangle_{D_{+}} \omega_{\beta}\left(\sigma(W)_{C_{-}}\left(\xi_{-}\right) W_{D_{-}}\left(\theta_{-}\right)\right) x_{A^{\prime}}(\theta) C^{A^{\prime} A} L_{s\left(I_{+}, I_{-}\right)}(\theta)_{A}^{D},
\end{aligned}
$$

where $X=W^{n}[x]$ and $Y=W^{m}[y]$ are two elements of $\mathcal{F}_{\beta}(S)$. Using the $T$-invariance of $\omega_{\beta}$ it is clear that $(,)_{\omega}$ has a unique extension to all of $\mathcal{F}_{\beta}(S)$. Singularities encountered for $\left(\omega_{-}\right)_{j}=\left(\theta_{-}\right)_{k}$ are declared in the sense of the principle value. Lemma B2 implies that $(,)_{\omega}$ is well defined, contravariant with respect to $\sigma$ and hermitian, i.e. satisfies (2.29). Further it has the announced property (2.28). For generic $\omega_{\beta}$ however $(,)_{\omega}$ is not positive semi-definite. 


\section{References}

[1] O. Babelon, D. Bernard and F.A. Smirnov, Comm. Math. Phys. 178 (1996) 281; 186 (1997) 601.

[2] H. Babujian, M. Karowski and A. Zapetal, J. Phys. A 30 (1997) 6425.

[3] R.J. Baxter, Exactly Solved Models in Statistical Mechanics (Academic Press, New York, 1982).

[4] D. Bernard and A. LeClair, Nucl. Phys. B 399 (1993) 709.

[5] J. Bisognano and E. Wichmann, J. Math. Phys. 16 (1975) 985; 17 (1976) 303.

[6] V. Brazhnikov and S. Lukyanov, Angular quantization and form factors in massive integrable models, hep-th/9707091.

[7] I. Cherednik, Comm. Math. Phys. 150 (1992) 109.

[8] B. Davies, Infinite-dimensional symmetry of corner transfer matrices, hep-th/9312141.

[9] H.J. De Vega, H. Eichenherr and J.M. Maillet, Comm. Math. Phys. 92 (1984) 507.

[10] C. Destri and H.J. De Vega, Nucl. Phys. B 406 (1993) 566.

[11] J. Ding: Hopf algebra extension of a ZF-algebra and its double, q-alg/9612008.

[12] C. Efthimiou and A. LeClair, Comm. Math. Phys. 171 (1995) 531.

[13] P.I. Etingof, I.B. Frenkel and A.A. Kirillov, Spherical functions on affine Lie groups, hep-th/940747.

[14] L. Faddeev, Sov. Sci. Rev. Math. Phys. C1 (1980) 107.

[15] I.B. Frenkel and N.Yu. Reshetikin, Comm. Math. Phys. 146 (1992) I.

[16] M. Golenishcheva-Kutuzova and V. Kac, $\Gamma$-conformal algebras; q-alg/9709006.

[17] R. Haag, W. Hugenholtz and M. Winnink, Comm. Math. Phys. 5 (1967) 215.

[18] K. Iohara, Bosonic representations of Yangian double, q-alg/9603033.

[19] M. Jimbo and T. Miwa, Algebraic analysis of solvable lattice models, CBMS Regional Confererence Series in Mathematics, Vol. 85 (AMS, 1994).

[20] M. Karowski and P. Weisz, Nucl. Phys. B 139 (1978) 455.

[21] S. Khoroshkin, D. Lebedev and S. Pakuliak, Intertwining operators for the central extension of the Yangian double, q-alg/9602030.

[22] S. Khoroshkin, D. Lebedev and S. Pakuliak, Lett. Math. Phys. 41 (1997) 31.

123] M. Lashkevich, JHEP 10 (1997) 003; hep-th/9704148.

[24] B.H. Lian and G.J. Zuckerman. Commutative quantum operator algebras, J. Pure Appl. Alg. 100 (1995) 117.

[25] A. Liguori, M. Mintchev and M. Rossi, J. Math. Phys. 38 (1997) 2888.

|26] S. Lukyanov, Comm. Math. Phys. 167 (1995) 183.

[27] M. Niedermaier, A derivation of the cyclic form factor equation, hep-th/9706172, Commun. Math. Phys., to appear.

[28| M. Niedermaier, Nucl. Phys. B 440 (1995) 603; B 456 (1995) 755 (E).

[29] M. Niedermaier, unpublished.

[30] I. Ojima, Ann. Phys. 137 (1981) 1.

[31) K.-H. Rehren, Spin-statistics and CPT for solitons, hep-th/9711085.

[32] N. Reshetikhin, Lett. Math. Phys. 26 (1992) 153.

[33] F.A. Smirnov, J. Phys. A 19 (1986) L575.

[34] F.A. Smirnov, Form factors in completely integrable models of quantum field theory (World Scientific, Singapore, 1992).

[35] F.A. Smirnov, Nucl. Phys. B 453 (1995) 807.

[36] F.A. Smirnov, Comm. Math. Phys. 155 (1993) 459.

[37] F.A. Smirnov, Int. J. Mod. Phys. A 7 Suppl. IB (1992) 813.

| $38 \mid$ B. Schroer, Nucl. Phys. B 499 (1997) 519

[39] H.B. Thacker, Physica D 18 (1986) 348.

|40| M. Takesaki, Tomita's theory of modular Hilbert algebras and its application, Lecture Notes in Mathematics (Springer, Berlin, 1970).

1411 V. Tarasov and A. Varchenko, Amer. Math. Soc. Transl. (2) 174 (1996) 235; hep-th/9406060.

|42| H. Umezawa, Advanced Field Theory (American Institute of Physics, 1993).

[43] A.B. Zamolodchikov and Al.B. Zamolodchikov, Ann. Phys. 120 (1979) 253. 